\title{
SEMICHARACTERISTICS, BORDISM, AND FREE GROUP ACTIONS
}

\author{
JAMES F. DAVIS AND R. JAMES MILGRAM
}

\begin{abstract}
In this paper we give characteristic class formulae for all semicharacteristic classes of all compact, closed manifolds with finite fundamental groups. These invariants are identified with elements in certain odd $L$-groups, and exactly which elements occur is specified. An appendix calculates the cohomology of the model groups needed. A second appendix determines the structure of the $L$-groups needed.
\end{abstract}

The Euler characteristic of an odd-dimensional manifold is zero; a natural substitute is a semicharacteristic-an alternating sum of the homology up to the middle dimension. Study of semicharacteristics was initiated by Kervaire [K] who examined their role in differential topology and geometry. The first semicharacteristic bordism invariant was introduced by DeRham. It turns out that

$$
\hat{\chi}_{1 / 2}\left(M^{2 k+1} ; K\right)=\sum_{i=1}^{k}(-1)^{i} \operatorname{dim}\left(H_{i}(M ; K)\right)
$$

is dependent on the characteristic of the field $K$ and is not a bordism invariant. However, if $k$ is even then the difference

$$
D R(M)=\hat{\chi}_{1 / 2}(M ; \mathbb{Q})-\hat{\chi}_{1 / 2}\left(M ; F_{2}\right) \bmod 2
$$

is an invariant of the oriented bordism group $\Omega_{2 k+1}$. Characteristic class formulae for this invariant were given in [L-M-P]. Equivariant bordism invariants were introduced by Lee [L] and studied by Stong [S]. If a group $G$ acts on an orientable manifold $M$, define the orientation character

$$
w: G \rightarrow\{ \pm 1\},
$$

by $w(g)=+1$ if and only if $g$ is orientation-preserving. An action of $G$ with orientation character $w$ is called a $(G, w)$-action. Let $\Omega_{n}(G, w)$ denote the bordism group of closed oriented manifolds with a free $(G, w)$-action; an element is zero if it is the boundary of a compact manifold with a free $(G, w)$ action. If $G$ is a finite group acting on a manifold $M$ of dimension $2 k+1$

Received by the editors Received by the editors November 23, 1987.

1980 Mathematics Subject Classification. Primary 57R67, 55N22, 55R40, 57Q20; Secondary 55M35, 55N25, 57N65, 57R20.

Research of the first author supported by an NSF Posdoctoral Fellowship.

Research of the second author supported by an NSF grant. 
and $K$ is a field of characteristic prime to $|G|$, then let

$$
\chi_{1 / 2}(M ; K)=\sum_{i=0}^{k}(-1)^{i}\left[H_{i}(M ; K)\right] \in K_{0}(K G),
$$

where $K_{0}(K G)$ is the Grothendieck group of virtual representations of $G$. Lee defines a homomorphism

$$
\begin{gathered}
\chi_{1 / 2}: \Omega_{n}(G, w) \rightarrow L^{n}(K G, w), \\
(M) \rightarrow \chi_{1 / 2}(M ; K)
\end{gathered}
$$

where $L^{n}(K G, w)$ is an appropriately defined subquotient of $K_{0}(K G)$. (Definitions are reviewed in §1.) The aim of this paper is to calculate this map completely and give characteristic class formulae for the map.

This problem also arises naturally from the point of view of modern surgery theory. Ranicki's symmetric signature $[R]$ gives a map

$$
\sigma^{*}: \Omega_{n}(G, w) \rightarrow L^{n}(\mathbb{Z} G, w)
$$

If one composes this with the natural change of rings map

$$
L^{n}(\mathbb{Z} G, w) \rightarrow L^{n}(K G, w)
$$

the composite can be identified with $\chi_{1 / 2}$ for $n$ odd. In fact this map factors through $L^{n}(\mathbb{Z}[s] G, w)$ where $\mathbb{Z}[s]$ is the polynomial ring with the involution given as $s \rightarrow 1-s$. The philosophy of $[\mathrm{M}-\mathrm{O}, \mathrm{M}]$, is that while $L^{n}(\mathbb{Z} G, w)$ represents the universal algebraic bordism invariants, $L^{n}(\mathbb{Z}[s] G, w)$ represents the universal computable algebraic bordism invariants, at least for $G$ a finite group. In accordance with that philosophy this paper completes the analysis of $L^{n}(\mathbb{Z}[s] G, w)$ (see [M-O] and Appendix 2 of this paper) and the image of $\Omega_{n}(G, w)$ (see $[\mathrm{M}]$, and the theorems below).

Our main results can be stated as follows.

Theorem A. Let $G$ be a finite group with orientation character $w$. Then, if

$$
n \equiv 3 \quad(\bmod 4)
$$

the map $\chi_{1 / 2}$ is identically zero. If $n \equiv 1(\bmod 4)$ then the image of $\chi_{1 / 2}$ is equal to that of

$$
\bigoplus i_{*}: L^{n}\left(\mathbb{Q} C,\left.w\right|_{C}\right) \rightarrow L^{n}(\mathbb{Q} G, w)
$$

where the sum is over all cyclic subgroups of $C$ of $G$.

\section{Theorem B.}

(i)

$$
\bigoplus \pi_{*}: \chi_{1 / 2}\left(\Omega_{n}(G, 1)\right) \rightarrow \bigoplus L^{n}(\mathbb{Q}[\mathbb{Z} / 2], 1)
$$

is injective where the sum is over all surjections $\pi: G \rightarrow \mathbb{Z} / 2$.

(ii) Let $\mathbb{Z} / 2 \times \mathbb{Z} / 4^{-}$denote the group $\mathbb{Z} / 2 \times \mathbb{Z} / 4$ with orientation character $w(1,0)=1, w(0,1)=-1$. Then if $w \neq 1$,

$$
\bigoplus \pi_{*}: \chi_{1 / 2}\left(\Omega_{n}(G, w)\right) \rightarrow \bigoplus L^{n}\left(\mathbb{Q}\left[\mathbb{Z} / 2 \times \mathbb{Z} / 4^{-}\right]\right)
$$


is injective where the sum is over all surjections $\pi: G \rightarrow \mathbb{Z} / 2 \times \mathbb{Z} / 4$ such that the orientation character of $G$ factors through the map $\mathbb{Z} / 2 \times \mathbb{Z} / 4 \rightarrow\{ \pm 1\}$ given above.

Thus we need only analyze the cases of $(G, w)=(\mathbb{Z} / 2,1)$ or $\mathbb{Z} / 2 \times \mathbb{Z} / 4^{-}$. In each case $L^{n} \cong \mathbb{Z} / 2$ for $n \equiv 1(\bmod 4)$. (For $(G, w)=(\mathbb{Z} / 2,1)$ we can identify $\chi_{1 / 2}(M ; \mathbb{Q})$ with $\hat{\chi}_{1 / 2}(M ; \mathbb{Q}) \in \mathbb{Z} / 2$.) The computation of $\chi_{1 / 2}$ in these cases is easily deduced from the work of Dupont and Lusztig [D-L]. The result is

Theorem C. Let $M^{n}$ represent an element of $\Omega_{n}(G, w), n \equiv 1(\bmod 4)$ and

denote the classifying map.

$$
f: M / G \rightarrow B_{G}
$$

(i) If $(G, w)=(\mathbb{Z} / 2,1)$ and $e \in H^{1}\left(\mathbb{Z} / 2 ; F_{2}\right)$ represents a generator then

$$
\chi_{1 / 2}(M ; \mathbb{Q})=\left\langle w_{n-1}(M / G) \cup f^{*}(e),[M / G]\right\rangle,
$$

where $w_{n-1}(M / G)$ is the $(n-1)$ th Stiefel-Whitney class of $M$.

(ii) If $(G, w)=\mathbb{Z} / 2 \times \mathbb{Z} / 4^{-}$and $\pi_{1}: \mathbb{Z} / 2 \times \mathbb{Z} / 4 \rightarrow \mathbb{Z} / 2$ denotes the projection, then $\chi_{1 / 2}(M ; \mathbb{Q})=\left\langle w_{n-1}(M / G) \cup\left(B_{\pi_{1}} \circ f\right)^{*}(e),[M / G]\right\rangle$.

As an example if $n \equiv 1(\bmod 4)$ then $L^{n}\left(\mathbb{Q}\left[(\mathbb{Z} / 2)^{m}\right], 1\right) \cong(\mathbb{Z} / 2)^{2^{m-1}}$ but the image of the semicharacteristic is $(\mathbb{Z} / 2)^{m}$. Thus the possible semicharacteristics are quite limited. If $m=2$, we recover the result that $\mathbb{Z} / 2 \times \mathbb{Z} / 2$ cannot act freely on $M^{n}$ if $\hat{\chi}_{1 / 2}(M, \mathbb{Q})$ is odd.

We conjecture that the three theorems above remain true if one replaces "closed manifold" by "Poincare duality complex", but we cannot prove this at this time.

The method of proof of the theorems above is similar to that of the "oozing conjecture" [M, M1, M2, H-M-T-W]. One plays off the fact that $\Omega_{n}(G, w)$ is a generalized homology theory, with induction theory results about $L^{n}(K G)$. First an easy transfer argument (see [S]) allows one to reduce to 2-groups. Then the induction theory of [H-T-W] allows one to reduce to certain special 2-groups, the $w$-model groups. The cohomology of these groups is calculated in Appendix 1 , allowing a further reduction to particular 2-groups. Then a second application of induction theory and the cohomology calculations allows one to reduce to the two special cases of Theorem $\mathrm{C}$ above, which have to be analyzed separately.

In a sequel to this paper we will apply our results to study free actions of elementary 2-groups on products of spheres. We completely determine the rational representations which arise from a free action on a manifold having the rational homology of a product of spheres.

\section{Preliminaries}

Our goal is to study the semicharacteristic bordism invariant defined for $n$ odd,

$$
\chi_{1 / 2}: \Omega_{n}(G ; w) \rightarrow L^{n}(\mathbb{Q} G, w) .
$$


In this section we give the definition of $\chi_{1 / 2}$, develop the bordism theory we need and discuss the algebraic computation of the $L$-groups.

Definition 1.1. Let $n=2 k+1$ be odd and $K$ a field of characteristic prime to $|G|$. If $V$ is a finitely generated $K G$-module, $V^{*}=\operatorname{Hom}_{K}(V, K)$ is given the structure of a $K G$-module by $(g \cdot \phi)(v)=w(g) \phi\left(g^{-1} v\right)$. Define $L^{n}(K G, w)$ to be the free abelian group with

Generators. $[V]$, which are the isomorphism classes of finitely generated $K G$ modules $V$ so that $V \cong V^{*}$.

Relations.

(i) $[V \oplus W]=[V]+[W]$.

(ii) $[V]=0$ if there is a KG-isomorphism $f: V \rightarrow V^{*}$ so that

$$
f=(-1)^{k+1} f^{*}: V\left(=V^{* *}\right) \rightarrow V^{*} .
$$

(iii) $[K G]=0$.

Remarks. (i) More generally, if $A$ is a semisimple ring with involution, the group $L_{h}^{n}(A)$ can be shown to be the group with generators and relations as above. These groups have exponent 2 since $V \oplus V^{*} \cong 2 V$ admits the standard symmetric or skew-symmetric hyperbolic form. Here $K G$ is given the involution $\tau\left(\sum a_{g} g\right)=\sum w(g) a_{g} g^{-1}$.

(ii) R. Lee [L] defined groups $R_{S p, K}(G, w)$ and $R_{O, K}(G, w)$. The groups $L^{n}(K G, w)$ inject to these groups when $n \equiv 1$ or $3(\bmod 4)$ respectively.

(iii) All $L$-groups in this paper are the $L_{h}$ or $L^{h}$-groups, unless otherwise indicated.

Definition 1.2. Given an action of $G$ on $M^{2 k+1}$ with orientation character $w$ one defines the surgery semicharacteristic

$$
\chi_{1 / 2}(M ; K)=\sum_{i=0}^{k}(-1)^{i}\left[H_{i}(M ; K)\right] \in L^{2 k+1}(K G, w) .
$$

In the case of a free action we will also use the notation $\chi_{1 / 2}(M / G ; K G)$.

R. Lee [L] showed that this gives a well-defined bordism invariant. From the viewpoint of modern surgery theory this map is obtained by taking the symmetric signature of $M$ in $L_{h}^{n}(\mathbb{Z} G)$, then pushing it forward to $L_{h}^{n}(K G)$ and then identifying this group with $L^{n}(K G, w)$ and the image of the symmetric signature with the surgery semicharacteristic (see [D-R] for example).

The group $\Omega_{n}(G, w)$ has (at least) three geometric interpretations. First, as above, it is the bordism group of closed oriented $n$-manifolds with free $G$ action and orientation character $w$. Alternatively, let $\zeta$ be the pullback of the canonical line bundle $\gamma$ over $B_{\mathbf{Z} / 2}$, 


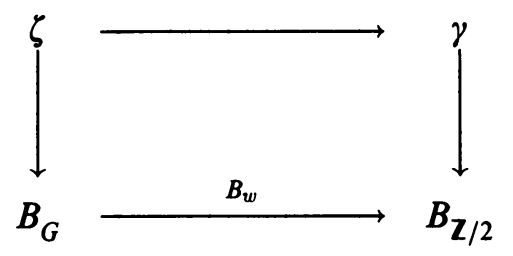

Then elements of $\Omega_{n}(G, w)$ are represented by closed $n$-manifolds $M^{n}$ with a map $f: M \rightarrow B_{G}$ and an orientation of $\tau_{M} \oplus f^{*} \zeta$ where $\tau_{M}$ is the tangent bundle of $M$. Finally $f: M \rightarrow B_{G}$ gives a map of pairs

$$
g:\left(D\left(f^{*}(\zeta)\right)\right), S\left(f^{*}(\zeta)\right) \rightarrow(D(\zeta), S(\zeta)) .
$$

This induces an isomorphism

$$
\Omega_{*}(G, w) \cong \Omega_{*+1}(D(\zeta), S(\zeta)),
$$

where the inverse map is given by making a map $f:(W, \partial W) \rightarrow(D(\zeta), S(\zeta))$ transverse to the zero section and considering $f: f^{-1}\left(B_{G}\right) \rightarrow B_{G}$. This third interpretation allows us to use standard facts from oriented bordism theory.

Let $i: H \rightarrow G$ be an inclusion of groups. There is a commutative diagram

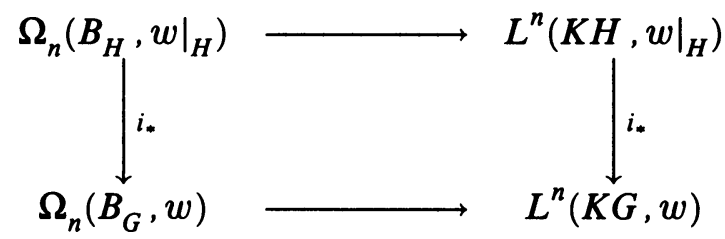

where $i_{*}(V)=K G \otimes_{K H} V$ and $i_{*}(M)=G \times_{H} M=G \times M /(g h, m) \sim$ $(g, h m)$. Here $\{g\} \times M$ has the negative orientation if $w(g)=-1$. There are also restriction maps $i^{*}$ going in the opposite direction defined by restricting the $G$-action to an $H$-action.

Now, suppose $i: G_{2} \rightarrow G$ is the inclusion of a 2-Sylow subgroup of the group $G$. Then standard transfer arguments based on the fact that $M S O_{(2)}$ is a wedge of Eilenberg-Mac Lane spectra show that

$$
i_{*} \circ i^{*}: \Omega_{n}(G, w)_{(2)} \rightarrow \Omega_{n}(G, w)_{(2)}
$$

is multiplication by $\left|G: G_{2}\right|$ (see [S] for more details). Since $L^{n}(G, w)$ has exponent two for $n$ odd, one deduces

Proposition 1.3. Let $G$ act freely on $M$ with orientation character $w$. Then

$$
\chi_{1 / 2}(M ; K)=i_{*}\left(\chi_{1 / 2}\left(i^{*}(M) ; K\right)\right) .
$$

We next review the algebraic computation of $L_{h}^{n}(A)$ for a semisimple ring (see also [D]). Let

$$
A=A_{1} \times \cdots \times A_{m}
$$

where the $A_{i} \cong M_{n_{i}}\left(D_{i}\right)$ and the $D_{i}$ are division rings. If $A_{i}$ is not invariant under the involution $\tau$ one says $A_{i}$ has type $G L$. If

$$
\operatorname{dim}_{K}\left(A_{i}\right) \text { is }>,=\text {, or }<2 \cdot \operatorname{dim}_{K}\left\{\alpha \in A_{i} \mid \tau(\alpha)=\alpha\right\}
$$


then we say that $A_{i}$ has type $S p, U$, or $O$ respectively. Let $V_{i}$ be an irreducible $A_{i}$-module; the type of $V_{i}$ is defined to be that of $A_{i}$. Then $L^{n}(A)$ is a $\mathbb{Z} / 2$-module generated by all $V_{i}$ so that $A_{i}$ has type $S p, U$, or $O$ subject to the following relations:

(i) $\left[V_{i}\right]=0$ if $D_{i}$ is noncommutative,

(ii) $\left[V_{i}\right]=0$ if $A_{i}$ has type $U$,

(iii) $\left[V_{i}\right]=0$ if $A_{i}$ has type $O$ and $n \equiv 3(\bmod 4)$ or if $A_{i}$ has type $S p$ and $n \equiv 1(\bmod 4)$

(iv) $\sum\left[V_{i}\right]=0$ where the sum is over all $i$ such that $n_{i}$ is odd.

In the case of a group ring there are character formulae which give the type. If $\chi$ is the character associated with an irreducible representation $V$ then $V$ is not of type $G L$ if and only if $\chi(g)=w(g) \chi\left(g^{-1}\right)$ for all $g \in G$. In particular $\rho: G \rightarrow G L(V)$ has type $G L$ if $w$ restricted to $\operatorname{ker}(\rho)$ is nontrivial. If $V$ does not have type $G L$ then it has type $O, U$, or $S p$ depending on whether $\sum w(g) \chi\left(g^{2}\right)$ is equal to $|G|, 0$, or $-|G|$. If $F$ is an extension field of $K$, $L^{n}(K G) \rightarrow L^{n}(F G)$ is injective.

In the case of the group ring $\mathbb{R} G$, when $w=1$ we have the classical results of Frobenius-Schur. No simple factor $A$ has type $G L ; A$ has type $O, U$, or $S p$ according to whether $A$ is real unramified $\left(A \cong M_{k}(\mathbb{R})\right), A$ is complex $\left(A \cong M_{k}(\mathbb{C})\right)$, or $A$ is real ramified $\left(A \cong M_{k}(\boldsymbol{H})\right)$. Thus $L^{3}(\mathbb{R} G)=0$. Since the type of a factor is preserved under extension of scalars (except for the case of a type $U$ factor which may become type $G L)$ one also has $L^{3}(K G)=0$ for any subfield $K$ of $\mathbb{R}$. (An alternate proof of this fact can be sketched as follows: Let $V$ be a $K G$-module. Choose a positive definite symmetric form over $K$ on $V$. Average over the group to make it equivariant. This form gives a symmetric isomorphism $V \rightarrow V^{*}$ and hence $V$ is trivial in $L^{3}(K G)$.)

Example 1.4. The group ring $\mathbb{Q}[\mathbb{Z} / 2] \cong \mathbb{Q}^{2}$ and each $\mathbb{Q}$ factor is type $O$. Thus

$$
L^{1}(\mathbb{Q}[\mathbb{Z} / 2], 1) \cong \mathbb{Z} / 2
$$

with the invariant being $\operatorname{Dim}_{\mathrm{Q}} V$. If $M^{4 k+1}$ is a closed manifold with an orientation preserving involution, then $\chi_{1 / 2}(M ; \mathbb{Q}) \in L^{4 k+1}(\mathbb{Q}[\mathbb{Z} / 2], 1)$ is given by the ordinary rational semicharacteristic $\hat{\chi}_{1 / 2}(M ; \mathbb{Q})$. The nontrivial element of the $L$-group is realized geometrically by the antipodal involution on $S^{4 k+1}$ with quotient $\mathbb{R P}^{4 k+1}$.

Example 1.5. Let $D_{2 p}$ denote the dihedral group of order $2 p$ with $p$ an odd prime. Then

$$
\mathbb{Q} D_{2 p}=\mathbb{Q} \times \mathbb{Q} \times M_{2}\left(\mathbb{Q}\left[\lambda_{p}\right]\right)
$$

where $\lambda_{p}=\zeta_{p}+\zeta_{p}^{-1}$ and $\zeta_{p}$ is a primitive $p$ th root of 1 . Let $V$ denote the irreducible representation associated to the matrix factor. All representations have type $O$ so $L^{1}\left(\mathbb{Q} D_{2 p}, 1\right) \cong(\mathbb{Z} / 2)^{2}$ with generators [Q] and $[V]$. If 
$i: \mathbb{Z} / 2 \rightarrow D_{2 p}$ is the inclusion of the 2-Sylow subgroup, then $i_{*}[\mathbb{Q}]=[\mathbb{Q}]+[V] \in$ $L^{1}\left(\mathbb{Q} D_{2 p}, 1\right)$. If $D_{2 p}$ acts freely preserving the orientation on a closed manifold $M^{4 k+1}$ then by 1.3 and 1.4 ,

$$
\chi_{1 / 2}(M ; \mathbb{Q})=\hat{\chi}_{1 / 2}(M ; \mathbb{Q}) \cdot([\mathbb{Q}]+[V])
$$

Thus we have reproved a result of Davis-Weinberger (see [D]).

Proposition 1.6. If $D_{2 p}$ acts freely on a closed oriented manifold of dimension $4 k+1$ and trivially on $H_{*}(M ; \mathbb{Q})$, then the rational semicharacteristic of $M$ is even. In particular $D_{2 p}$ cannot act freely on a rational homology sphere of dimension $4 k+1$.

\section{ABELIAN GROUPS}

For any cyclic group $\mathbb{Z} / n$ there is a faithful representation $\mathbb{Z} / n \rightarrow$ $\mathbb{Q}\left(\zeta_{n}\right)$ where $\zeta_{n}$ is a primitive $n$th root of unity. A surjection of a group $\phi: G \rightarrow \mathbb{Z} / n$ thus gives an irreducible representation $\phi: G \rightarrow \mathbb{Q}\left(\zeta_{n}\right)$. For $G$ abelian all representations arise in this way:

$$
\mathbb{Q} G \cong \prod_{\phi \in \mathscr{S}(G, \mathscr{B})} \mathbb{Q}\left(\zeta_{|\phi(G)|}\right)
$$

where $\mathscr{S}(G, \mathscr{C})$ is the set of surjections of $G$ onto a cyclic group.

Let $G$ be a group with orientation character $w: G \rightarrow\{ \pm 1\}$. Let $\phi: G \rightarrow \mathbb{Z} / n$ be a surjection. We have

TABLE 2.1

\begin{tabular}{|l|l|}
\hline \multicolumn{1}{|c|}{$n$} & \multicolumn{1}{c|}{ type of $\mathbf{Q}\left(\zeta_{n}\right)$} \\
\hline 1 or 2 & $\begin{array}{l}O \text { if } w=1 \\
G L \text { if } w \neq 1\end{array}$ \\
\hline 4 & $\begin{array}{l}O \text { if }(-1)^{\phi(g)}=w(g) \text { all } g \in G \\
U \text { if } w=1 \\
G L \text { otherwise }\end{array}$ \\
\hline $2^{k}, k>2$ & $\begin{array}{l}U \text { if } \operatorname{ker}(\phi) \subset \operatorname{ker}(w) \\
G L \text { otherwise }\end{array}$ \\
\hline
\end{tabular}

To figure out characteristic class formulae for the semicharacteristic there are two key cases,

$$
\begin{gathered}
G=\mathbb{Z} / 2 \quad(w=1), \\
G=\mathbb{Z} / 2 \times \mathbb{Z} / 4^{-} \quad \text { with } w(1,0)=1, w(0,1)=-1 .
\end{gathered}
$$

Example 2.2. Let $\mathbb{Z} / 2 \times \mathbb{Z} / 4^{-}$be as above, then

$$
\mathbb{Q}\left[\mathbb{Z} / 2 \times \mathbb{Z} / 4^{-}\right] \cong \mathbb{Q}^{4} \times \mathbb{Q}[i]^{2},
$$

each $\mathbb{Q}$-factor has type $G L$ and each $\mathbb{Q}[i]$-factor has type $O$. Thus

$$
L^{1}\left(\mathbb{Q}\left[\mathbb{Z} / 2 \times \mathbb{Z} / 4^{-}\right]\right) \cong \mathbb{Z} / 2,
$$


represented by either one of the $\mathbb{Q}[i]$-factors. The nontrivial element is realized geometrically, in fact the composition

$$
\boldsymbol{\Omega}_{4 k+1}(\mathbb{Z} / 2) \rightarrow \boldsymbol{\Omega}_{4 k+1}\left(\mathbb{Z} / 2 \times \mathbb{Z} / 4^{-}\right) \rightarrow L^{4 k+1}\left(\mathbb{Q}\left[\mathbb{Z} / 2 \times \mathbb{Z} / 4^{-}\right]\right)
$$

is onto. Indeed if $S^{4 k+1}$ is the sphere with the free antipodal $\mathbb{Z} / 2$-action we have

$$
\chi_{1 / 2}\left(S^{4 k+1} \times \mathbb{Z} / 4^{-} ; \mathbb{Q}\right) \cong\{\mathbb{Q} \times \mathbb{Q} \times \mathbb{Q}[i]\}
$$

In both key cases characteristic class formulae can be deduced from a result of Dupont and Lusztig [D-L, 2.7]:

Theorem 2.3. Let $M$ be a connected closed manifold of dimension $4 k+1$ with $w_{1}(M)^{2}=0$. Let $h: M \rightarrow B_{\mathbf{Z} / 2}$ be a map with $\xi: \widetilde{M} \rightarrow M$ the induced $\mathbb{Z} / 2$ cover and $e \in H^{1}\left(B_{\mathbf{Z} / 2} ; F_{2}\right)$ the generator. Let $\mathbb{C}^{\Gamma}$ be the complex numbers given the structure of $a \pi_{1}(M)$-module via a homomorphism $\Gamma: \pi_{1}(M) \rightarrow \mathbb{C}^{\times}$ whose image has order 1, 2, or 4. Then

$$
\hat{\chi}_{1 / 2}\left(\widetilde{M} ; \mathbb{C}^{\Gamma \circ \xi_{*}}\right)=\left\langle w_{4 k}(M) \cup h^{*}(e),[M]\right\rangle .
$$

Corollary 2.4. Let $\left(M, f: M \rightarrow B_{G}\right) \in \Omega_{4 k+1}(G, w)$.

(i) If $(G, w)=(\mathbb{Z} / 2,1)$ then

$$
\chi_{1 / 2}(M ; \mathbb{Q} G)=\left\langle w_{4 k}(M) \cup f^{*}(e),[M]\right\rangle .
$$

(ii) If $(G, w)=\mathbb{Z} / 2 \times \mathbb{Z} / 4^{-}$and $\pi_{1}: \mathbb{Z} / 2 \times \mathbb{Z} / 4 \rightarrow \mathbb{Z} / 2$ is the projection, then

$$
\chi_{1 / 2}(M ; \mathbb{Q} G)=\left\langle w_{4 k}(M) \cup\left(B_{\pi_{1}} \circ f\right)^{*}(e),[M]\right\rangle .
$$

Proof. For part (i) simply take $h=f$ and $\Gamma$ to be the trivial map. Part (i) could also be deduced from the work of Stong [S].

For part (ii) take $h=B_{\pi_{1}} \circ f$ and let $\Gamma=\pi_{2} \circ f_{*}: \pi_{1}(M) \rightarrow \mathbb{Z} / 4 \hookrightarrow$ $\mathbb{C}^{\times}$. Then $\hat{\chi}_{1 / 2}\left(\widetilde{M} ; \mathbb{C}^{\Gamma \circ \xi_{*}}\right)$ gives the number of $\mathbb{Q}[i]$-summands occurring in $\chi_{1 / 2}(M ; \mathbb{Q} G)$.

With these basic building blocks we can compute characteristic class formulae for any abelian 2-group $G$ with orientation character $w$. Write

$$
G=G_{1} \times \cdots \times G_{n}
$$

as a product of cyclic groups with $w\left(G_{i}\right)=1$ for $i<n$. Let $\pi_{i}: G \rightarrow \mathbb{Z} / 2$ denote the composite of the projection onto the $i$ th factor with the surjection $G_{i} / 2 G_{i} \cong \mathbb{Z} / 2$. Table 2.1 shows

\section{Proposition 2.5.}

(i) $L^{3}(\mathbb{Q} G, w)=0$ for any $w$.

(ii) $L^{1}(\mathbb{Q} G, 1) \cong L^{1}(\mathbb{Q}[G / 2 G], 1) \cong \bigoplus_{\phi \in \mathcal{P}(G, \mathbf{Z} / 2)} L^{1}(\mathbb{Q}[\mathbb{Z} / 2], 1) \cong(\mathbb{Z} / 2)^{2^{n}-1}$, where $\mathscr{S}(G, \mathbb{Z} / 2)$ denotes the set of surjections of $G$ onto $\mathbb{Z} / 2$.

(iii) If $w \neq 1$ and $\left|G_{n}\right|=2$ then $L^{1}(\mathbb{Q} G, w)=0$. 
(iv) If $w \neq 1$ and $\left|G_{n}\right|>2$ then

$$
\begin{aligned}
L^{1}(\mathbb{Q}, w) & \cong L^{1}\left(\mathbb{Q}\left[G_{1} / 2 G_{1} \times \cdots \times G_{n-1} / 2 G_{n-1} \times G_{n} / 4 G_{n}\right], w\right) \\
& \cong \bigoplus_{\phi \in \mathscr{S}\left(G_{1} \times \cdots \times G_{n-1}, \mathbb{Z} / 2\right)} L^{1}\left(\mathbb{Q}\left[\mathbb{Z} / 2 \times \mathbb{Z} / 4^{-}\right]\right) \cong(\mathbb{Z} / 2)^{2^{n-1}-1} .
\end{aligned}
$$

Any $\phi \in \mathscr{S}(G, \mathbb{Z} / 2)$ can be expressed as a sum

$$
\phi=a_{1}(\phi) \pi_{1}+\cdots+a_{n}(\phi) \pi_{n}
$$

where the indices $a_{i}(\phi)$ are 0 or 1 , and not all zero. Let $(M, f) \in \Omega_{4 k+1}(G, 1)$. The characteristic class formula for $\phi_{*}\left(\chi_{1 / 2}(M ; \mathbb{Q} G)\right) \in L^{1}(\mathbb{Q} G, 1) \cong \mathbb{Z} / 2$ is given by

$$
\sum a_{i}(\phi)\left\langle w_{4 k}(M) \cup\left(B_{\pi_{i}} \circ f\right)^{*}(e),[M]\right\rangle .
$$

On the other hand, if $M_{i}$ is a lens space with fundamental group $G_{i}$, and the classifying map for the universal covering is $M_{i} \rightarrow B_{C_{i}}$ and $f_{i}$ is the composite

$$
M_{i} \rightarrow B_{G_{i}} \stackrel{B_{i n c}}{\rightarrow} B_{G}
$$

then $\left\langle w_{4 k}\left(M_{i}\right) \cup\left(B_{\pi_{j}} \circ f_{i}\right)^{*}(e),\left[M_{i}\right]\right\rangle=\delta_{i j}$. Thus

$$
\chi_{1 / 2}\left(\Omega_{4 k+1}(G, 1)\right) \cong(\mathbb{Z} / 2)^{n}
$$

with a basis given by the $M_{i}$ above. In particular this shows that the possible homology of manifolds of dimension $4 k+1$ equipped with a free orientation preserving action of an abelian group is quite restricted.

A way of rephrasing the above is that for elementary 2 -groups $G$, the semicharacteristics that arise are precisely those in the kernel of all restriction maps

$$
i^{*}: L^{1}(\mathbb{Q} G, 1) \rightarrow L^{1}(\mathbb{Q} H, 1)
$$

where $H$ is a proper subgroup of $G$.

In the case where $w \neq 1$ and $\left|G_{n}\right|>2$, an analysis similar to the previous case can be given. In this case the image of the semicharacteristic map is isomorphic to $(\mathbb{Z} / 2)^{n-1}$ with a basis given by the $\left(M_{i}, f_{i}\right), i=1,2, \ldots, n-1$.

For general abelian groups $G \cong G_{2} \times G_{\text {odd }}$ it is easily shown that

$$
L^{2 n+1}(\mathbb{Q} G, w) \cong L^{2 n+1}\left(\mathbb{Q} G_{2},\left.w\right|_{G_{2}}\right)
$$

so that no new information can be gained.

Example 2.7. For $G=\mathbb{Z} / 2 \times \mathbb{Z} / 2$ it was previously known that for

$$
(M, f) \in \Omega_{4 k+1}(G, 1),
$$

the ordinary rational semicharacteristic was zero $(\bmod 2)$. (So, for example, $\mathbb{Z} / 2 \times \mathbb{Z} / 2$ cannot act freely on any rational homology sphere.) We see this by restricting to any proper $\mathbb{Z} / 2$-subgroup of $G$. Indeed, it was a desire to fully understand this phenomenon which motivated the current paper. 
Following Davis-Weinberger [D], we deduce

Theorem 2.8. Let $G$ be a finite group acting freely on a closed orientable manifold $M^{4 k+1}$ whose ordinary rational semicharacteristic is odd. If $G$ acts trivially on $H_{i}(M ; \mathbb{Q})$ for $i \leq 2 k$, then $G$ is the product of a cyclic 2-group and a group of odd order.

Proof. First note that the action must be orientation preserving. Indeed if $f$ is an orientation-reversing map, then the Lefschetz number of $f$ is twice the semicharacteristic.

Since $w=1$, we already know by 1.6 and 2.9 , that $G$ is not equal to $D_{2 p}$ ( $p$ an odd prime) or $\mathbb{Z} / 2 \times \mathbb{Z} / 2$. If we set $\mathscr{C}$ to be the largest class of finite groups such that

(i) $H \subset G \in \mathscr{C} \Rightarrow H \in \mathscr{C}$,

(ii) $G \in \mathscr{C}, H$ normal in $G \Rightarrow G / H \in \mathscr{C}$,

(iii) $D_{2 p}, \mathbb{Z} / 2 \times \mathbb{Z} / 2$ do not belong to $\mathscr{C}$,

then $\mathscr{C}$ is the set of groups which are the product of a cyclic 2-group and a group of odd order (see [D]). The theorem follows.

\section{REVIEW OF INDUCTION THEORY}

In this section we use induction theory to show that the $L$-groups are detected by certain special groups, the $w$-model groups.

The rational representation theory of 2-groups is quite limited

Definition 3.1. The basic 2-groups are: cyclic groups $\quad \mathbb{Z} / 2^{n}$,

dihedral groups $\quad D_{2^{n}}=\left\langle x, y \mid x^{2^{n-1}}=y^{2}=1, y x y^{-1}=x^{-1}\right\rangle, n \geq 4$, semidihedral groups $S D_{2^{n}}=\left\langle x, y \mid x^{2^{n-1}}=y^{2}=1, y x y^{-1}=x^{2^{n-2}-1}\right\rangle, n \geq 4$, quaternionic groups $Q_{2^{n}}=\left\langle x, y \mid x^{2^{n-2}}=y^{2}, y x y^{-1}=x^{-1}\right\rangle, n \geq 3$.

There is a unique faithful representation for each of the groups above. Their central role in representation theory is explained by

Theorem 3.2 (Roquette-Fontaine). Let $\rho: G \rightarrow G L(V)$ be an irreducible rational representation of a finite 2-group $G$. Then there is a sequence of subgroups

$$
H_{0} \subset H_{1} \subset \cdots \subset H_{k}=G
$$

each of index 2 in the next with representations $\rho_{i}: H_{i} \rightarrow G L\left(V_{i}\right)$ such that

(a) $V_{i+1}$ is induced from $V_{i}$,

(b) $\operatorname{End}_{\mathrm{QH}_{i}}\left(V_{i}\right) \cong \operatorname{End}_{\mathrm{QH} H_{i+1}}\left(V_{i+1}\right)$,

(c) $\rho_{k}=\rho$,

(d) $H_{0} / \operatorname{ker}\left(\rho_{0}\right)$ is a basic group.

When one worries about the type of the representation one has to be a little more careful. The situation is fairly easy when $w=1$, namely the type of $V_{0}$ is the same as the type of $V_{k}$. This is because the type can be read off of 
$D_{i}=\operatorname{End}_{\mathbf{Q H}_{i}}\left(\operatorname{Ind}\left(V_{i}\right)\right) ; V_{i}$ is type $\mathrm{O}, \mathrm{U}$, or Sp depending on whether $D_{i} \otimes_{\mathbf{Q}} \mathbf{R}$ is a product of factors isomorphic to $M_{n}(\mathbf{R}), M_{n}(\mathbb{C})$, or $M_{n}(\mathbf{H})$ respectively.

If $N$ is a normal subgroup of a subgroup $H$ of $G$ and $w$ is trivial on $N$, there is a map

$$
L^{2 n+1}(\mathbb{Q} G, w) \rightarrow L^{2 n+1}(\mathbb{Q}[H / N], w)
$$

given by first restricting to $H$ and then inducing from $H$ to $H / N$.

Theorem 3.3 (Oriented induction theorem). Let $G$ be a finite 2-group. Then

$$
L^{2 n+1}(\mathbb{Q} G) \rightarrow \bigoplus L^{2 n+1}(\mathbb{Q}[H / N])
$$

is injective where the sum is over all $N \subset H \subset G$ with $N$ normal in $H$ and $H / N$ isomorphic to a basic group.

The proof is analogous to [H-T-W, Theorem 0.19].

For the case of $w \neq 1$ we need results of [H-T-W].

Definition 3.4. Let $w: G \rightarrow\{ \pm 1\}$ be a homomorphism. $(G, w)$ is $w$-basic if all abelian subgroups of $\operatorname{ker}(w)$ which are normal in $G$ are cyclic.

Lemma 3.5 [H-T-W]. If $G$ is a 2-group then $(G, w)$ is $w$-basic if and only if $G$ is basic or $G$ is given as a semidirect product

$$
1 \rightarrow \operatorname{ker}(w) \rightarrow G \rightarrow\{ \pm 1\} \rightarrow 1
$$

where $\operatorname{ker}(w)$ is basic and the twisting is determined by a homomorphism

$$
f: \operatorname{ker}(w) \rightarrow \mathbb{Z} / 2=\{0,1\}
$$

together with an element $c \in \operatorname{ker}(f)$ which is central in $\operatorname{ker}(w)$, and has order 2. The twisting $\alpha:\{ \pm 1\} \rightarrow \operatorname{Aut}(\operatorname{ker}(w))$ is given by $\alpha(-1)(g)=g c^{f(g)}$.

The corresponding quadratic induction theorem is

Theorem 3.6 [H-T-W]. Let $\rho: G \rightarrow G L(V)$ be an irreducible rational representation of a finite 2-group $G$ with orientation character $w: G \rightarrow\{ \pm 1\}$. Then there is a sequence of subgroups

$$
H_{0} \subset H_{1} \subset \cdots \subset H_{k}=G
$$

each of index 2 in the next one with representations $\rho_{i}: H_{i} \rightarrow G L\left(V_{i}\right)$ such that

(a) $V_{i+1}$ is induced from $V_{i}$,

(b) $\operatorname{End}_{\mathrm{QH} H_{i}}\left(V_{i}\right) \cong \operatorname{End}_{\mathrm{QH}}\left(V_{i+1}\right)$,

(c) $\rho_{k}=\rho$,

(d) $H_{0} /\left(\operatorname{ker}\left(\rho_{0} \cap \operatorname{ker}(w)\right)\right.$ is a w-basic group and the type of $V_{0}$ as a representation of this group is the same as the type of $V$.

This does not mean that the $w$-basic groups suffice to detect $L^{2 n+1}(\mathbb{Q} G, w)$. For example, $\mathbb{Z} / 2 \times \mathbb{Z} / 4^{-}$is not $w$-basic and $L^{1}\left(\mathbb{Q}\left[\mathbb{Z} / 2 \times \mathbb{Z} / 4^{-}\right]\right)$is not detected by any proper subquotients. 
However, we do have

Theorem 3.7. Suppose $G$ is a finite 2-group with orientation character $w$. Then we have an injection

$$
L^{2 n+1}(\mathbb{Q} G, w) \rightarrow L^{2 n+1}(\mathbb{Q}[H / N], w)
$$

where the sum is over

(a) all $w$-basic subquotients so that $w$ is trivial on $N$,

(b) all $(H / N, w) \cong \mathbb{Z} / 2 \times \mathbb{Z} / 4^{-}$,

(c) all $(H / N, w) \cong\left(\left(\mathbb{Z} / 2 \times \mathbb{Z} / 4^{-}\right) \odot \mathbb{Z} / 2, w\right)$ where the generator $g$ of the new $\mathbb{Z} / 2$ acts on $\mathbb{Z} / 2 \times \mathbb{Z} / 4^{-}$by $g(1,0) g^{-1}=(1,2), g(0,1) g^{-1}=(0,1)$ and $w(g)=1$.

Remark. We denote the semidirect product described in $3.7(\mathrm{c})$ by the symbol $M_{16}$, and discuss its homology in Appendix A.1.2. The $w$-basic groups together with the groups in (b) and (c) we call the $w$-model groups. The cohomology of the $w$-model groups is computed in Appendix 1 , and a table of their types is given in Appendix 3.

3.7 is a formal consequence of the following two facts:

(A) There is an exact sequence

$$
\mathbb{Z} / 2 \stackrel{\alpha_{2 n+1}}{\rightarrow} L_{K}^{2 n+1}(\mathbb{Q} G, w) \rightarrow L_{h}^{2 n+1}(\mathbb{Q} G, w) \rightarrow 0
$$

and $L_{K}^{2 n+1}\left(A_{1} \times A_{2}\right) \cong L_{K}^{2 n+1}\left(A_{1}\right) \times L_{K}^{2 n+1}\left(A_{2}\right)$.

(B) $\alpha_{3}$ is trivial and $\alpha_{1}$ is injective if and only if $w$ factors through the surjection

$$
\mathbb{Z} / 4 \rightarrow\{ \pm 1\}
$$

The proof of 3.7 then follows as in [H-T-W, 0.20] where an analogous case is considered.

For a semisimple ring A with involution we define $L_{K}^{2 n+1}(A)$ just as in Definition 1.1 except for leaving out relation (iii). One defines $\alpha_{2 n+1}(1)=[\mathbb{Q} G]$. Then $\alpha_{3}$ is trivial because $\mathbb{Q} G$ admits a nonsingular symmetric form.

$\alpha_{1}$ is injective precisely when $\mathbb{Q} G$ does not admit a nonsingular skewsymmetric form. A simple factor of $\mathbb{Q} G$ which is not commutative admits a nonsingular skew-symmetric form so we need only concentrate on abelian representations. But by our analysis in $\S 2$, the abelianization $G^{a b}$ has a type $O$ representation if and only if $w$ factors through $\mathbb{Z} / 4$.

\section{The general CASE}

For an abelian group $A$, let $A^{w}$ be the $\mathbb{Z} G$-module given by $(g, a) \rightarrow w(g) a$. For the case of general $G$ it becomes convenient to rephrase the semicharacteristic homomorphism in terms of "index" homomorphisms

$$
I_{j}: H_{j}\left(G, \mathbb{Z}_{(2)}^{w \prime}\right) \rightarrow L^{n}(\mathbb{Q} G, w)
$$


natural with respect to induction and transfer maps. Here $\mathrm{n}$ is odd and we set $I_{j}=0$ if $n-j$ is not divisible by 4 . For any $\left(M, f: M \rightarrow B_{G}\right) \in \Omega_{n}(G, w)$ we have

$$
\chi_{1 / 2}(M ; \mathbb{Q} G)=\sum I_{j}\left(f_{*}\left\langle\left(V_{2 *}\right)^{2}\left(\tau_{M} \oplus f^{*} \zeta\right) \cap[M]\right\rangle\right) .
$$

Here $V_{2 *}=1+v_{2}+v_{4}+\cdots$ is the even part of the total Wu-class of $M$ and $\zeta$ is the line bundle given by $w$.

Remark. This is a close relative of the formula for the symmetric signature given by Taylor-Williams (see [T-W, 1.1, 1.5]). They derive their formula from Ranicki's factorization of the symmetric signature map

$$
\Omega_{*}(G, w)=H_{*}(G, w ; M S O) \rightarrow H_{*}\left(G, w ; \mathbb{L}^{0}(\mathbb{Z})\right) \rightarrow L^{*}(\mathbb{Z} G, w)_{(2)} .
$$

The notation $H_{*}(G, w ; M S O)$ is defined in [R]; by the Thom isomorphism theorem it is $\tilde{H}_{*+1}(T(\zeta) ; M S O)$. Taylor-Williams construct cohomology classes $L \in H^{4 *}\left(\mathbf{L}^{0}(\mathbb{Z}) ; \mathbb{Z}_{(2)}\right)$ and $r \in H^{4 *+1}\left(\mathbb{L}^{0}(\mathbb{Z}) ; \mathbb{Z} / 2\right)$ which exhibit $\mathbb{L}^{0}(\mathbb{Z})_{(2)}$ as a wedge of Eilenberg-Mac Lane spectra and thus give maps

$$
\begin{gathered}
I_{j}: H_{j}\left(G ; \mathbb{Z}_{(2)}^{w}\right) \rightarrow L^{j+4 k}(\mathbb{Q} G, w)_{(2)}, \\
r_{j}: H_{j}(G ; \mathbb{Z} / 2) \rightarrow L^{j+4 k+1}(\mathbb{Q} G, w)_{(2)} .
\end{gathered}
$$

One sees that maps of the second type vanish since there is a factorization

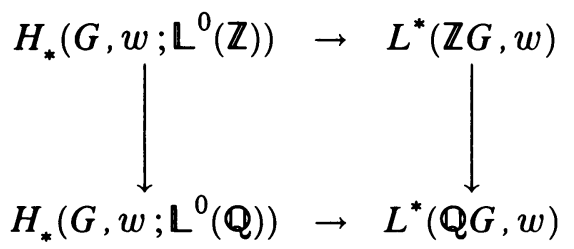

and $L^{j}(\mathbb{Q})=0$ for $j$ not divisible by 4 . The cohomology class formula 4.1 follows from the fact that if $\sigma: M S O \rightarrow \mathbb{L}^{0}$ is the usual map then the Thom isomorphism applied to $\sigma^{*}(L)$ is $\left(V_{2 *}\right)^{2} \in H^{*}\left(B_{S O} ; \mathbb{Z} / 2\right)$. Notice that $\mathrm{n}$ is odd so we only need to use $\mathbb{Z} / 2$-coefficients.

The key result of this paper can then be stated as follows:

Theorem 4.2. (a) For any group $G$ and for any orientation character $w$ of $G$, $I_{j}=0$ for $j>1$.

(b) For $(G, w)=\mathbb{Z} / 2$ or $\mathbb{Z} / 2 \times \mathbb{Z} / 4^{-}, I_{1}$ is an isomorphism.

Proof. Since $\tau_{M} \oplus f^{*} \zeta$ is oriented,

$$
\left(V_{2 *}\right)^{2}\left(\tau_{M} \oplus f^{*} \zeta\right)=V^{2}\left(\tau_{M} \oplus f^{*} \zeta\right)=V^{2}\left(\tau_{M}\right) f^{*} V^{2}(\zeta) .
$$

For $(M, f) \in \Omega_{4 k+1}(G, w)$ and $(G, w)=\mathbb{Z} / 2$ or $\mathbb{Z} / 2 \times \mathbb{Z} / 4^{-}, f^{*} V^{2}(\zeta)=1$, so the computation of the $I_{j}$ 's follows from 4.1 and 2.4.

To prove the vanishing of the $I_{j}$ 's for $j>1$ for a general group $G$ we use a series of reductions. Whenever one has a generation theorem in homology 
or a detection theorem in $L$-theory, one can reduce the proof of (a) to smaller groups.

Step 1. It suffices to prove (a) for $G$ a 2-group. (This is because

$$
H_{*}\left(G_{2} ; \mathbb{Z}_{(2)}^{w}\right) \rightarrow H_{*}\left(G ; \mathbb{Z}_{(2)}^{w}\right)
$$

is onto.)

Step 2. It suffices to prove (a) for the $w$-model groups. (This follows immediately from the detection theorem.)

Step 3. It suffices to prove (a) for $(G, w)=(\mathbb{Z} / 2,1)$ or $\mathbb{Z} / 2 \times \mathbb{Z} / 4^{-}$.

Indeed if $G$ is a $w$-model group then either

(i) $G$ has no faithful rational representations of the form $M_{2 i}(K)$ for some field $K$, having type $O$ or $S p$, in which case $L^{2 k+1}(\mathbb{Q} G, w)$ is detected by smaller groups, or

(ii) the odd-dimensional homology is generated by proper subgroups by 4.3 below, or

(iii) $G$ is abelian in which case $L^{2 k+1}(\mathbb{Q} G, w)$ is detected by $(\mathbb{Z} / 2,1)$ or $\mathbb{Z} / 2 \times \mathbb{Z} / 4^{-}$.

Lemma 4.3. For any $G$ which is a $w$-model group with faithful rational representation of the form $M_{2^{i}}(K)$ for some field $K$, having type $O$ or $S p$, then

$$
\coprod_{i} H_{*}\left(H_{i} ; \mathbb{Z}^{w}\right) \rightarrow H_{*}\left(G ; \mathbb{Z}^{w}\right)
$$

is surjective in odd dimensions where the sum is over all subgroups $H_{i} \subset G$ which are abelian, generalized quaterionic, or the product of $\mathbb{Z} / 2$ with a generalized quaternionic group.

(Lemma 4.3 is proved as Theorem A.1.0.3 in Appendix 1. In Theorem A.1.0.2 we prove a stronger result for homology with untwisted coefficients. However in the generality of twisted coefficients A.1.0.3 is sharp.)

Remark. A complete list of $w$-model groups and their faithful representations is given in the table in Appendix 3.

Knowledge of the index homomorphism determines characteristic class formulae as in $\S 2$ and conversely. Indeed the index homomorphism factors through homology with $\mathbb{Z} / 2$-coefficients

$$
I_{1}: H_{1}(G ; \mathbb{Z} / 2) \rightarrow L^{n}(\mathbb{Q} G, w)
$$

Choose a basis $\left\{B_{i}\right\}$ for the $L$-group. Then, using this basis to identify the $L$-group with its dual

$$
\operatorname{Hom}\left(L^{n}(\mathbb{Q} G, w), \mathbb{Z} / 2\right),
$$

and dualizing the above map one gets

$$
I_{1}^{*}: L^{n}(\mathbb{Q} G, w) \rightarrow H^{1}(G ; \mathbb{Z} / 2)
$$


The characteristic class formula is then given by

$$
\chi_{1 / 2}(M ; \mathbb{Q} G)=\sum\left\langle\left(V_{2 *}\right)^{2}(M) \cup f^{*}\left(I_{1}^{*}\left(B_{i}\right),[M]\right\rangle\left[B_{i}\right] .\right.
$$

Example 4.4. Let $(G, w)=\left(D_{2^{i}}, 1\right)$. Then $L^{n}(\mathbb{Q} G, 1) \cong(Z / 2)^{i+1}$ with generators given by $\left[\mathbb{Q}_{+-}\right],\left[\mathbb{Q}_{-+}\right],\left[\mathbb{Q}_{--}\right]$, and the $\left[W_{j}\right]$ where $\phi: G \rightarrow W_{j}$ is an irreducible representation satisfying $G / \operatorname{ker}(\phi) \cong D_{2^{j}}, j=3, \ldots, i$. Here $\mathbb{Q}_{+-}$(for example) refers to the representation $G \rightarrow \mathbb{Q}$ where $g \rightarrow 1, T \rightarrow-1$. Then $H_{1}$ is generated by elements $[g],[T]$. Since we know $I_{1}$ on cyclic subgroups,

$$
\begin{gathered}
I_{1}\left(D_{2^{i}}\right)([g])=i_{*}\left(I_{1}\langle g\rangle\right)([g])=i_{*}\left[\mathbb{Q}_{-}\right]=\left[\mathbb{Q}_{-+}\right]+\left[\mathbb{Q}_{--}\right], \\
I_{1}\left(D_{2^{i}}\right)([T])=i_{*}\left(I_{1}(\langle T\rangle)([T])=i_{*}\left[\mathbb{Q}_{-}\right]=\left[\mathbb{Q}_{+-}\right]+\left[\mathbb{Q}_{--}\right]+\sum\left[W_{j}\right] .\right.
\end{gathered}
$$

If we write $H^{1}\left(D_{2^{i}} ; \mathbb{Z} / 2\right)=(\mathbb{Z} / 2) e_{i}+(\mathbb{Z} / 2) e_{2}$ where $\left\{e_{1}, e_{2}\right\}$ is the dual basis to $\{[g],[T]\}$, then for $(M, f) \in \Omega_{4 k+1}\left(D_{2^{i}}, 1\right)$ we have

$$
\begin{aligned}
\chi_{1 / 2}(M ; \mathbb{Q} G)= & \left\langle V^{2}(M) \cup f^{*}\left(e_{1}\right),[M]\right\rangle\left[\mathbb{Q}_{-+}\right] \\
& +\left\langle V^{2}(M) \cup f^{*}\left(e_{2}\right),[M]\right\rangle\left[\mathbb{Q}_{+-}\right] \\
& +\left\langle V^{2}(M) \cup f^{*}\left(e_{1}+e_{2}\right),[M]\right\rangle\left[\mathbb{Q}_{--}\right] \\
& +\left\langle V^{2}(M) \cup f^{*}\left(e_{2}\right),[M]\right\rangle \sum\left[W_{j}\right] .
\end{aligned}
$$

\section{APPENDIX 1}

THE COHOMOLOGY OF THE MODEL 2-GROUPS

When $\pi$ is a finite 2-group, there is a correspondence between irreducible $(w$-twisted) representations and subquotients

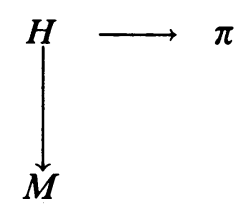

where $M$ runs over certain limited families of model groups. These are the Fontaine models for $w$ trivial, and the " $w$-basic groups" of Taylor-Williams for $w: \pi \rightarrow \mathbb{Z} / 2$ nontrivial. (However, for $L$-theory reasons we examine the $w$-model groups, which are the $w$-basic groups together with two extra groups $M_{16}$, and $\mathbb{Z} / 2 \times \mathbb{Z} / 4^{-}$, which we study in $\S A .1 .2$. In this appendix we calculate $H^{*}\left(M ; F_{2}\right)$ as $M$ runs over the model groups above. (The mod-2 cohomology of $w$-model groups which are metacyclic has been computed before, see [Mu, $\mathrm{Di}, \mathrm{Ru}]$.) In particular, one result of the calculation is

Theorem A.1.0.1. Let $M$ be a w-model group as above with $M \neq W_{2^{i+1}}$, and $\alpha \in H^{j}\left(M ; F_{2}\right)$ then there is a subgroup $V \subset M$ so that $r^{*}(\alpha) \in H^{j}\left(V ; F_{2}\right)$ is 
nonzero. Moreover $V$ is abelian, a quaternion group $Q_{2^{i+1}}, i \geq 2$, or $\mathbb{Z} / 2 \times$ $Q_{2^{i+1}}$.

(Here, $W_{2^{i+1}}$ is a specific model group-and one of the simplest nontrivial ones-which is defined and studied in $\S A .1 .2$ of this appendix. Moreover, the only classes which are needed from $W_{2^{i+1}}$ in terms of representing bordism classes which could carry nontrivial semicharacteristic classes are one twodimensional class and one 5-dimensional class.)

We actually need results for the integral homology of certain twisted $w$-model groups.

Theorem A.1.0.2. $H^{*}(G ; \mathbb{Z}) \rightarrow \amalg_{H \subset G} H^{*}(H ; \mathbb{Z})$ is a split injection for $(G, w)$ any $w$-model group except $W_{2^{i}}$, where $H \subset G$ runs over all abelian and generalized quaternion subgroups, or has the form $\mathbb{Z} / 2 \times Q_{2^{i}}$.

Theorem A.1.0.3. For any $G$ which is a w-model group with faithful rational representation of Schur index one having type $O$ or $S p$, then

$$
\coprod_{i} H_{*}\left(H_{i} ; \mathbb{Z}^{w}\right) \rightarrow H_{*}\left(G ; \mathbb{Z}^{w}\right)
$$

is surjective in ODD dimensions where the sum is over all subgroups $H_{i} \subset G$ which are abelian, generalized quaternionic, or the product of $\mathbb{Z} / 2$ with a generalized quaternion group.

(These two results will be proved in $\S$ A.1.6.)

\section{A.1.1. Calculational techniques.}

Let $C \triangleleft G \rightarrow H \rightarrow 1$ be a central extension, then, as is well known, this extension is classified by an element $K(G) \in H^{2}(H ; C)$. Actually, there is a fibration sequence

$$
K(C, 1) \rightarrow B_{G} \rightarrow B_{H} \stackrel{\alpha}{\rightarrow} K(C, 2)
$$

with $\alpha^{*}(l)=K(G) \in H^{2}\left(B_{H} ; C\right)$ determining the fibration. Here $K(C, 1)$ and $K(C, 2)$ are Eilenberg-Mac Lane spaces. This gives rise to a Leray-Serre spectral sequence with $E_{2}$ term $E_{2}^{i, j}=H^{i}\left(B_{H} ; H^{j}\left(B_{C} ; F_{2}\right)\right)$ converging to $H^{*}\left(B_{G} ; \mathbb{F}_{2}\right)$. The $d^{2}$ differential is determined by $K(G)$. For example, if $C=\mathbb{Z} / 2$, then $B_{C}=B_{\mathbf{Z} / 2}$ and

$$
E_{2}^{i, j}=H^{i}\left(B_{H} ; \mathbb{F}_{2}\right) \otimes H^{j}\left(B_{\mathbf{Z} / 2} ; \mathbb{F}_{2}\right)=H^{i}\left(B_{H} ; \mathbb{Z}_{2}\right) \otimes \mathbb{F}_{2}[\kappa]
$$

while $d^{2}(\kappa)=K(G) \in H^{2}\left(B_{H} ; \mathbb{F}_{2}\right)$. In case the ideal

$$
I=(K(G)) \subset H^{*}\left(B_{H} ; F_{2}\right)
$$

is free over $H^{2}\left(B_{H} ; \mathbb{F}_{2}\right)$ then $E_{3}=H^{*}\left(B_{H} ; F_{2}\right) / I \otimes F_{2}\left[\kappa^{2}\right]$ and $d^{3}, d^{5}$ are determined by $d^{3}\left(\kappa^{2}\right)=S q^{1}(K(G)), d^{5}\left(\kappa^{4}\right)=S q^{2} S q^{1}(K(G))$, from the Serre transgression theorem.

This procedure will, in the examples, be almost entirely sufficient. In particular, in all cases, the formula above will show that $d^{5}=0$, and $E_{5}=$ 
$E_{\infty}$. But in at least one case, after calculating $H^{*}\left(B_{G} ; \mathrm{F}_{2}\right)$ we will need more delicate information to enable us to understand the various restriction maps $r_{i}: H^{*}\left(B_{G} ; F_{2}\right) \rightarrow H^{*}\left(B_{V_{i}} ; F_{2}\right)$ where the $V_{i} \subset G$ run over all index 2 subgroups.

Lemma A.1.1.1. There is a 1-1 correspondence between line bundles on $B_{G}$, nonzero elements in $H^{1}\left(B_{G} ; F_{2}\right)$ and index 2 subgroups $V_{i} \subset G$.

(Line bundles are classified by maps into $\mathbf{R P}^{\infty}=K(\mathbb{Z} / 2,1)$. Thus, the set of line bundles is equivalent to the set $\operatorname{Hom}(G, \mathbb{Z} / 2)$, and this set, in turn, is equivalent under the correspondence $f \rightarrow \operatorname{Ker}(f)$ to the set of index 2 subgroups of $G$.)

Indeed, the line bundles are just the (open) mapping cylinders of the 2-fold covering maps $B_{V_{i}} \rightarrow B_{G}$.

Theorem A.1.1.2. Let $V \subset G$ be an index 2 subgroup, let $e \in H^{1}\left(G ; F_{2}\right)$ correspond to the line bundle, then there is an exact sequence

$$
. . \rightarrow H^{*}\left(B_{V} ; \mathrm{F}_{2}\right) \stackrel{t r^{*}}{\longrightarrow} \widetilde{H}^{*}\left(B_{G} ; \mathrm{F}_{2}\right) \stackrel{\cup e}{\rightarrow} H^{*+1}\left(B_{G} ; \mathrm{F}_{2}\right) \stackrel{r^{*}}{\rightarrow} H^{*+1}\left(B_{V} ; \mathrm{F}_{2}\right) \rightarrow . .
$$

(This is just a reinterpretation of the Gysin sequence in our case.)

Remark. Actually, the map $t r^{*}$ in the theorem is the transfer map, but we will not need this fact in what follows.

\section{A.1.2. Two examples.}

A key example in what follows is the group

$$
M_{16}=\left\{x, y, z \mid x^{4}=y^{2}=z^{2}=1, z y z^{-1}=x^{2} y, z x z^{-1}=x, y x=x y\right\} .
$$

It has a central extension of the form $1 \rightarrow \mathbb{Z} / 2 \rightarrow M_{16} \rightarrow(\mathbb{Z} / 2)^{3} \rightarrow 1$ and writing $H^{*}\left((\mathbb{Z} / 2)^{3} ; \mathbf{F}_{2}\right)=\mathbf{F}_{2}[a, b, c]$, the $K$-invariant becomes $a^{2}+b c$. So, additively,

$$
H^{*}\left(M_{16} ; \mathbf{F}_{2}\right)=\mathbf{F}_{2}\left[\kappa^{4}\right](1+a)\left(\mathbf{F}_{2}[b](1+b c)+\mathbf{F}_{2}[c]\right) .
$$

(Here $F_{2}[x]$ is the polynomial algebra on the generator $x$, so the expression above expands to $F_{2}\left[\kappa_{4}, b\right] \oplus \mathbf{F}_{2}\left[\kappa_{4}, b\right] a \oplus \mathbf{F}_{2}\left[\kappa_{4}, b\right] b c \oplus \mathbf{F}_{2}\left[\kappa_{4}, b\right] a b c \oplus$ $F_{2}\left[\kappa_{4}, c\right] c \oplus F_{2}\left[\kappa_{4}, c\right] a c$. This gives an additive basis for the cohomology ring, with some information on the cup product structure. The relations $a^{2}=b c$, $b^{2} c=b c^{2}$ now completely specify the multiplicative structure.)

Next, consider the 3 subgroups $\mathbb{Z} / 4 \times \mathbb{Z} / 2=V_{1}=V_{2}=V_{3} \subset M_{16}$ which are given as

$$
V_{1}=\{x, y\}, \quad V_{2}=\{x, z\}, \quad V_{3}=\{x, x y z\} \text {. }
$$

Then in cohomology

$$
\begin{aligned}
& \operatorname{Ker}\left(r_{1}^{*}\right)=F_{2}\left[\kappa^{4}\right](1+a)\left(F_{2}[b] b c+F_{2}[c] c\right) \\
& \operatorname{Ker}\left(r_{2}^{*}\right)=F_{2}\left[\kappa^{4}\right](1+a)\left(F_{2}[b] b+F_{2}[b] b c\right) \\
& \operatorname{Ker}\left(r_{3}^{*}\right)=F_{2}\left[\kappa^{4}\right](1+a)\left(F_{2}[b]\left(b^{2}+b c\right)+F_{2}[c](b+c)\right)
\end{aligned}
$$


But the intersection of these kernels is the ideal generated by $b c(b+c)$, which is 0 in $H^{*}\left(M_{16} ; F_{2}\right)$. It follows that these 3 subgroups detect the $\bmod (2)$ cohomology of $M_{16}$.

Example A.1.2.2. Consider the groups

$$
W_{2^{i+1}}=\mathbb{Z} / 2^{i} \odot_{\alpha} \mathbb{Z} / 2=\left\{x, y \mid x^{2^{i}}=y^{2}=1, y x y=x^{1+2^{i-1}}\right\} \text {. }
$$

We have a central extension $\mathbb{Z} / 2 \triangleleft W_{2^{i+1}} \longrightarrow \mathbb{Z} / 2^{i-1} \times \mathbb{Z} / 2$, and writing

$$
H^{*}\left(\mathbb{Z} / 2^{i-1} \times \mathbb{Z} / 2 ; \mathbb{F}_{2}\right)=E[a] \otimes \mathbb{F}_{2}[b, c],
$$

with $\operatorname{dim}(a)=\operatorname{dim}(c)=1, \operatorname{dim}(b)=2$, we have $K(W)=b+a c$.

Theorem A.1.2.4. $H^{*}\left(W_{2^{i+1}} ; \mathbb{F}_{2}\right)=E\left[e_{1}, e_{3}\right] \otimes F_{2}\left[c, \kappa^{4}\right] /\left(e_{1} c^{2}=0\right), \operatorname{dim}\left(e_{1}\right)$ $=\operatorname{dim}(c)=1, \operatorname{dim}\left(e_{3}\right)=3, \operatorname{dim}\left(\kappa^{4}\right)=4$, and

$$
S q^{1}\left(e_{3}\right)=S q^{1}\left(\kappa^{4}\right)=S q^{1}\left(e_{1}\right)=0, S q^{2}\left(e_{3}\right)=c^{2} e_{3}+e_{1} \kappa^{4} .
$$

Proof. Note that $S q^{1}(b+a c)=a c^{2}$. Using the spectral sequence of the central extension to calculate we then have $E_{5}^{*, *}=\mathbb{F}_{2}\left[\kappa^{4}\right]\left(1+a+a c+\left(1+a \kappa^{2}\right) \mathbb{F}_{2}[c]\right)$. The class represented by $a \kappa^{2}$ we call $e_{3}$ and the image of $a$ we call $e_{1}$. Hence $e_{1} c^{2}=0$. Restrict to the subgroup $\mathbb{Z} / 2^{i}=\{x\}$, then $e_{1} \rightarrow a, e_{3} \rightarrow a b, \kappa^{4} \rightarrow b^{2}$, $e_{1} e_{3} \rightarrow 0$, so $e_{1} e_{3}=m c e_{3}+n c^{4}, m, n \in \mathbb{F}_{2}$. From this $0=e_{1}^{2} e_{3}=m e_{1} c e_{3}+$ $n e_{1} c^{4}=m^{2} c^{2} e_{3}+m n c^{5}$, and it follows that $m=0$. Also, $0=\left(e_{1} e_{3}\right)^{2}=n^{2} c^{8}$ so $n=0$ as well. Thus $e_{1} e_{3}=0$.

Let $V \subset W_{2^{i+1}}$ be the subgroup $V=\mathbb{Z} / 2^{i-1} \times \mathbb{Z} / 2=\left\{x^{2}, y\right\}$, then writing

$$
H^{*}\left(V ; \mathbb{F}_{2}\right)=E[h] \otimes \mathbb{F}_{2}[d, f],
$$

with $\operatorname{dim}(d)=2$, we have

Lemma A.1.2.5. $r^{*}\left(e_{1}\right)=0, r^{*}(c)=f, r^{*}\left(\kappa^{4}\right)=d^{2}, r^{*}\left(e_{3}\right)=h f^{2}$.

Proof. The only thing which is not clear is $r^{*}\left(e_{3}\right)$. But we have the cofibration

$$
B_{V} \rightarrow B_{W_{2^{i+1}}} \rightarrow M\left(\zeta_{e_{1}}\right)
$$

and, since $\left(e_{1}\right)^{2}=0$, we have $\operatorname{tr}(h)=e$. Hence $\operatorname{tr}\left(f^{2} h\right)=c^{2} \operatorname{tr}(h)=c^{2} e=0$. From this $f^{2} h \in \operatorname{im}\left(r^{*}\right)$, and the only thing available to hit it is $e_{3}$.

Note that $r^{*}: H^{4}\left(W_{2^{i+1}} ; \mathrm{F}_{2}\right) \rightarrow H^{4}\left(V ; F_{2}\right)$ is an injection. This implies that $S q^{1}\left(e_{3}\right)=0$. Next, note that $\operatorname{ker}\left(r^{*}\right)$ in dimension 5 is a single copy of $F_{2}$ with generator $e_{1} \kappa^{4}$, and $S q^{1}\left(\kappa^{4}\right)=0$ since otherwise $e_{3}$ would be an infinite survivor in the Bockstein spectral sequence, which is impossible. Thus $S q^{1}\left(e_{1} \kappa^{4}\right)=0$ and $S q^{2}\left(e_{3}\right)=c^{2} e_{3}+m e_{1} \kappa^{4}, m \in \mathbf{F}_{2}$. Hence $\left(e_{3}\right)^{2}=S q^{3}\left(e_{3}\right)=$ 0 , as required.

Finally, restrict again to $\mathbb{Z} / 2^{i}$. Then, since $S q^{2}(a b)=a b^{2} \neq 0$ we see that $m$ above is 1 and we have $S q^{2}\left(e_{3}\right)=c^{2} \dot{e}_{3}+e_{1} \kappa^{4}$, completing the proof. 
Remark. For this group the class $e_{1} c$ is in the kernel of the map to all three of the index two subgroups. We have already verified this for $\{x\}$ and $\left\{x^{2}, y\right\}$. The remaining subgroup is $\left\{x^{2}, x y\right\}$ but $(x y)^{2}=x^{2+2^{i-1}}$ and so this last subgroup is $\mathbb{Z} / 2^{i}$ and the result is clear. In particular, $e_{1} c$ is not detected on any subgroup of $W_{2^{i+1}}$. Hence, also $e_{3} e_{1} c$ and these 2 elements multiplied by $F_{2}\left[\kappa^{4}\right]$ are not detected on any subgroups.

\section{A.1.3. The standard (Fontaine) model groups.}

These are the groups which carry the faithful irreducible representations which induce up from subquotients to give all irreducible rational representations on finite 2-groups. They are completely described in $\S 3.1$. Their $\bmod (2)$ cohomology is also well known. We have

Theorem A.1.3.1. $H^{*}\left(D_{2^{i+1}} ; \mathbf{F}_{2}\right)=\mathbf{F}_{2}[w] \otimes\left(\mathbf{F}_{2}[e]+F_{2}[f]\right)$, where $\operatorname{dim}(w)=2$, $\operatorname{dim}(e)=\operatorname{dim}(f)=1, e \cup f=0$, and under the correspondence

$$
\operatorname{Hom}(G ; \mathbb{Z} / 2)=H^{1}\left(B_{G} ; \mathbb{Z} / 2\right)
$$

we have $e(x)=1, e(y)=0$, while $f(x)=1, f(y)=1$. The detecting subgroups are two copies of $\mathbb{Z} / 2 \times \mathbb{Z} / 2,\left\langle x^{2^{i-1}}, y\right\rangle$, and $\left\langle x^{2^{i-1}}, x y\right\rangle$. In both cases the cohomology maps take $w$ to $A^{2}+A B$ where $A$ is dual to $x^{2^{i-1}}$.

Theorem A.1.3.2. $H^{*}\left(Q_{8} ; \mathrm{F}_{2}\right)=\mathrm{F}_{2}\left[\kappa^{4}\right]\left(1+a+b+a^{2}+b^{2}+a^{2} b\right)$, with relations

$$
a^{2}+b^{2}=a b, \quad a^{2} b=a b^{2},
$$

and

$$
H^{*}\left(Q_{2^{i}} ; \mathrm{F}_{2}\right)=\mathrm{F}_{2}\left[\kappa^{4}\right]\left(1+a+b+a^{2}+b^{2}+a^{3}\right) \text { for } i>3
$$

with relations $a^{3}=b^{3}$, and $a b=0$.

Theorem A.1.3.3. $H^{*}\left(S D_{2^{i+1}} ; \mathrm{F}_{2}\right)=\mathrm{F}_{2}\left[\kappa^{4}\right]\left(1+e+e^{2}+\left(1+e_{3}\right) \mathrm{F}_{2}[c]\right)$ where $e^{3}=0$. Here, $\operatorname{dim}(e)=\operatorname{dim}(c)=1$, and $\operatorname{dim}\left(e_{3}\right)=3$. The 3 index 2 subgroups of $S D_{2^{i+1}}$ are

$D_{2^{i}}=\left\{x^{2}, y\right\}$ with inclusion $r, Q_{2^{i}}=\left\{x^{2}, x y\right\}$ with inclusion $\bar{r}$,

$$
\mathbb{Z} / 2^{i}=\{x\} \text { with inclusion } \hat{r},
$$

and

$$
\begin{gathered}
r^{*}(e)=0, r^{*}(c)=(e+f), r^{*}\left(\kappa^{4}\right)=w^{2}, r^{*}\left(e_{3}\right)=(e+f) w, \\
\bar{r}^{*}(e)=a+b, \bar{r}^{*}(c)=0, \bar{r}^{*}\left(\kappa^{4}\right)=\kappa^{4} .
\end{gathered}
$$

In particular, the first two subgroups detect $H^{*}\left(S D_{2^{i+1}} ; \mathrm{F}_{2}\right)$.

Remark. The detection for $Q_{2^{i+1}}$ is more delicate. The elements $a^{2}, b^{2}, a b^{2}$ or $a^{3}$ are not detected on any subgroups, but $F_{2}\left[\kappa^{4}\right]$ is detected on the central $\mathbb{Z} / 2$. 


\section{A.1.4. The Taylor-Williams $w$-basic groups.}

In each of the model groups $M$ in the last section there is a unique central $\mathbb{Z} / 2$ with generator $c$. Given a homomorphism $f: M \rightarrow \mathbb{Z} / 2=\{0,1\}$ there is a central automorphism $\phi_{f}: M \rightarrow M$ defined by $\phi_{f}(g)=g c^{f(g)}$, and we define

$$
M_{f}=M \odot_{\phi_{f}} \mathbb{Z} / 2
$$

where, if $z$ generates the new $\mathbb{Z} / 2$, and $g \in M$ we have $z g z^{-1}=\phi_{f}(g)$, and $z^{2}=1$. These $M_{f}$, where $M$ runs over the previous model groups and $f$ over $\operatorname{Hom}(M, \mathbb{Z} / 2)$, are the Taylor-Williams $w$-basic groups.

Example. The group $W_{2^{i+1}}$ of $\S \mathrm{A} .1 .2$ is $\left(\mathbb{Z} / 2^{i}\right)_{f}$ where $f: \mathbb{Z} / 2^{i} \rightarrow \mathbb{Z} / 2$ is the surjection.

If $M=D_{2^{i+1}}$, then, up to isomorphism there are two distinct nontrivial homomorphisms $f_{i}: D_{2^{i+1}} \rightarrow \mathbb{Z} / 2$ given by

$$
\begin{aligned}
& f_{1}(x)=1, \quad f_{1}(y)=0, \\
& f_{2}(x)=0, \quad f_{2}(y)=1,
\end{aligned}
$$

which define $D_{2^{i+1}, f_{1}}, D_{2^{i+1}, f_{2}}$ associated to $D_{2^{i+1}}$. For the semidihedral groups $S D_{2^{i+1}}$ with $i \geq 3$ there are 3 distinct isomorphisms

$$
\begin{array}{ll}
f_{1}(x)=1, & f_{1}(y)=0, \\
f_{2}(x)=0, & f_{2}(y)=1, \\
f_{3}(x)=1, & f_{3}(y)=1 .
\end{array}
$$

For $Q_{8}$, up to isomorphism there is only one nontrivial $f, f(x)=1, f(y)=$ 0 , while for $Q_{2^{i+1}}$ with $i \geq 3$ there are two,

$$
\begin{array}{ll}
f_{1}(x)=1, & f_{1}(y)=0, \\
f_{2}(x)=0, & f_{2}(y)=1 .
\end{array}
$$

This set of groups can be reduced to three families since there are a number of isomorphisms of families above. Indeed, we have

\section{Lemma A.1.4.2.}

(a) $D_{2^{i+1}, f_{1}} \cong S D_{2^{i+1}, f_{1}}$.

(b) $D_{2^{i+1}, f_{2}} \cong S D_{2^{i+1}, f_{2}}$,

(c) $S D_{2^{i+1}, f_{3}} \cong Q_{2^{i+1}, f_{1}}, i \geq 3$,

(d) $D_{2^{i+1}, f_{2}} \cong Q_{2^{i+1}, f_{2}}, i \geq 2$.

Proof. We have the presentation (A.1.4.3)

$$
D_{2^{i+1}, f_{1}}=\left\{x, y, z \mid x^{2^{i}}=y^{2}=x^{2}=1, y z=z y, z x=x^{1+2^{i-1}} z, y x=x^{-1} y\right\}
$$

so $y z x y z=x^{2^{i-1}-1}$ and $z(y z) z=y z, z x z=x^{1+2^{i-1}}$. Thus $\{x, y z\} \cong S D_{2^{i+1}}$. Next $S D_{2^{i+1}} \subset D_{2^{i+1}, f_{2}}$ as $\{z x, y\}$. Similarly $Q_{2^{i+1}} \subset S D_{2^{i+1}, f_{3}}$ as $\{x, y z\}$, and 
$D_{2^{i+1}} \subset Q_{2^{i+1}, f_{2}}$ as $\{x, y z\}$. These embeddings extend to semidirect product embeddings by adjoining $z$ in all cases, and the lemma follows.

Finally, here is one more isomorphism which is very useful.

Lemma A.1.4.4. $M_{16} \cong D_{8, f_{2}}$.

Proof. In $D_{8, f_{2}}$ consider the generators $x z, y, z$. Clearly $x z=z x$, so

$$
(x z)^{2}=x^{2}, y z=(x z)^{2} z y, \text { and } y(x z) y=x^{3} y z y=x z .
$$

But these are the relations defining $M_{16}$, and the result follows since both groups have order 16.

\section{A.1.5. The cohomology of the Taylor-Williams $w$-basic groups.}

We begin with the groups $D_{2^{i+1}, f_{1}}$ and $D_{2^{i+1}, f_{2}}$. These are given as central extensions

$$
\mathbb{Z} / 2 \rightarrow D_{2^{i+1}, f_{i}} \rightarrow D_{2^{i}} \times \mathbb{Z} / 2
$$

with $K$-invariants $w+e h$ ( $h$ is dual to the generator $z$ ) for $f_{1}$ and for $f_{2}$ $w+(e+f) h$. We calculate directly using the spectral sequences for these extensions

$$
H^{*}\left(D_{2^{i+1}, f_{1}} ; \mathrm{F}_{2}\right)=\mathrm{F}_{2}\left[\kappa^{4}\right]\left(\mathrm{F}_{2}[e](1+e h)+\left(1+e_{3}\right) \mathrm{F}_{2}[f, h]\right)
$$

where $e_{3}$ is represented by $f \kappa^{2}$, and $e h^{2}=0$. Similaily,

$$
H^{*}\left(D_{2^{i+1}, f_{2}} ; \mathrm{F}_{2}\right)=\mathrm{F}_{2}\left[\kappa^{4}\right]\left((1+e h+f h)\left(\mathbf{F}_{2}[e]+\mathbf{F}_{2}[f]\right)+\mathbf{F}_{2}[h]\left(1+e h^{2}\right)\right) .
$$

Here $(e+f) h^{2}=0$ so $e^{2} h^{2}=f^{2} h^{2}=0$.

Lemma A.1.5.2. For $i \geq 4$ there are two embeddings of $D_{2^{i}, f_{2}}$ in $D_{2^{i+1}, f_{2}}$ given as

$$
\operatorname{im}\left(r_{1}\right)=\left\{x^{2}, y, z\right\}, \quad \operatorname{im}\left(r_{2}\right)=\left\{x^{2}, x y, z\right\},
$$

and

$$
r_{1}^{*}+r_{2}^{*}: H^{*}\left(D_{2^{i+1}, f_{2}} ; \mathbf{F}_{2}\right) \rightarrow H^{*}\left(D_{2^{i}, f_{2}} ; \mathbf{F}_{2}\right) \oplus H^{*}\left(D_{2^{i}, f_{2}} ; \mathbf{F}_{2}\right)
$$

is an injection.

Proof. $r_{1}^{*}(e)=0, r_{1}^{*}(f)=(e+f), r_{1}^{*}(h)=h, r_{1}^{*}\left(\kappa^{4}\right)=\kappa^{4}$. Similarly, $r_{2}^{*}(e)=e+f, r_{2}^{*}(f)=e+f, r_{2}^{*}(h)=h, r^{*}\left(\kappa^{4}\right)=\kappa^{4}$. From this the result follows.

Corollary A.1.5.3. $H^{*}\left(D_{2^{i+1}, f_{2}} ; \mathbf{F}_{2}\right)$ is detected by abelian subgroups of the form $\mathbb{Z} / 4 \times \mathbb{Z} / 2$.

(By iterating the previous lemma, we see that copies of $H^{*}\left(M_{16} ; F_{2}\right)$ detect, but the results of $\S A .1 .2$ show that the cohomology of this group is detected by groups of the form $\mathbb{Z} / 4 \times \mathbb{Z} / 2$.) 
Lemma A.1.5.4. The two embeddings $r_{1}: D_{2^{i}} \times \mathbb{Z} / 2 \rightarrow D_{2^{i+1}, f_{1}}$ as $\left\{x^{2}, y, z\right\}$ and $r_{2}: D_{2^{i}, f_{2}} \rightarrow D_{2^{i+1}, f_{1}}$ as $\left\{x^{2}, x y, z\right\}$ detect $H^{*}\left(D_{2^{i+1}, f_{1}} ; F_{2}\right)$.

(Indeed $r_{1}^{*}(h)=h, r_{1}^{*}(e)=0, r_{1}^{*}(f)=e+f, r_{1}^{*}\left(e_{3}\right)=(e+f) w, r_{1}^{*}\left(\kappa^{4}\right)=w^{2}$, while $r_{2}^{*}(h)=h, r_{2}^{*}(e)=e+f, r_{2}^{*}(f)=e+f, r_{2}^{*}\left(\kappa^{4}\right)=\kappa^{4}$. From this the result follows.)

Corollary A.1.5.5. $H^{*}\left(D_{2^{i+1}, f_{1}} ; \mathbb{F}_{2}\right)$ is detected by subgroups for the form $(\mathbb{Z} / 2)^{3}$ and $\mathbb{Z} / 4 \times \mathbb{Z} / 2$. In particular it is detected on abelian subgroups.

Next we consider the group $S D_{2^{i+1}, f_{3}}$. The central extension

$$
\mathbb{Z} / 2 \rightarrow S D_{2^{i+1}, f_{3}} \rightarrow D_{2^{i}} \times \mathbb{Z} / 2
$$

has $K$-invariant $e^{2}+w+f h$, and so

$$
H^{*}\left(S D_{2^{i+1}, f_{3}} ; \mathbb{F}_{2}\right)=\mathbb{F}_{2}\left[\kappa^{4}\right]\left(\mathbb{F}_{2}[h]\left(1+e+e^{2}+e^{3}\right)+(1+h f) \mathbb{F}_{2}[f]\right) .
$$

Note that $e^{4}=0,(h f)^{2}=0$.

Lemma A.1.5.7. $Q_{2^{i}} \times \mathbb{Z} / 2=\left\{x^{2}, x y, x\right\} \subset S D_{2^{i+1}, f_{3}}$ and $D_{2^{i}, f_{2}}=\left\{x^{2}, y, z\right\} \subset$ $S D_{2^{i+1}, f_{3}}$ detect $H^{*}\left(S D_{2^{i+1}, f_{3}} ; \mathbb{F}_{2}\right)$.

(This is routine. The first group detects the part $\mathbb{F}_{2}\left[\kappa^{4}\right] \mathbb{F}_{2}[h]\left(1+e+e^{2}+e^{3}\right)$ while the second detects $\mathbb{F}_{2}\left[\kappa^{4}\right] \mathbb{F}_{2}[f](1+h f)$. )

This completes the cohomology calculations for the groups we need.

\section{A.1.6 The integral results.}

In this section we first outline the proof of Theorem A.1.0.2, and then, in somewhat more detail sketch the proof of Theorem A.1.0.3. They are both fairly direct evaluations based on the use of the Bockstein spectral sequence. Our $\bmod (2)$ calculations in the previous sections give just enough information to calculate the $E_{2}$-terms in each case, and for some perhaps strange reason, they all basically look the same from $E_{2}$ on, at least in the untwisted case, while in the twisted case, though there are some unpleasant classes, none of them occur in odd dimensions. A good reference for the naturality, multiplicativity, and calculational properties of the Bockstein spectral sequence is [B]

Lemma A.1.6.1. Let $\phi: \bigsqcup_{H_{i} \subset G} B_{H_{i}} \rightarrow B_{G}$ be the maps of classifying spaces induced by the inclusions, and suppose

$$
\phi^{*}: H^{*}\left(B_{G}, \mathbb{Z} / 2\right) \rightarrow \coprod_{H_{i}} H^{*}\left(B_{H_{i}} ; \mathbb{Z} / 2\right)
$$

is injective, then if $V \subset H^{*}\left(B_{G} ; \mathbb{Z}\right) \otimes \mathbb{Z} / 2$ is the kernel of the map

$$
H^{*}\left(B_{G} ; \mathbb{Z}\right) \otimes \mathbb{Z} / 2 \rightarrow E_{2}\left(B_{G}\right)
$$


where $E_{2}(\quad)$ is the $E_{2}$-term of the Bockstein spectral sequence, then there is a subgroup $W \subset H^{*}\left(B_{G} ; \mathbb{Z}\right)$ with $2 W=0, p: W \rightarrow V$ an isomorphism, and

$$
W \rightarrow H^{*}\left(B_{G} ; \mathbb{Z}\right) \rightarrow \coprod_{H_{i}} H^{*}\left(B_{H_{i}} ; \mathbb{Z}\right)
$$

is a split injection.

Proof. $V=\operatorname{im}\left(S q^{1}\right)$. Note that if $\alpha \in V$ then $\alpha=S q^{1}(\beta)$ so we have that $S q^{1}\left(\phi^{*}(\beta)\right)=\phi^{*}(\alpha) \neq 0$, and the result follows.

Remark A.1.6.2. $W \subset H^{*}\left(B_{G} ; \mathbb{Z}\right)$ and $W \cong \operatorname{Kernel}(\cdot 2) / \operatorname{im}(\cdot 2)$.

Lemma A.1.6.3. For $(G, w)$ any $w$-basic group except $W_{2^{i}}$ or $\mathbb{Z} / 2$, we have that in the Bockstein spectral sequence $E_{2}\left(B_{G}\right)=E\left(e_{3}\right) \otimes F_{2}\left[\kappa_{4}\right]$.

Lemma A.1.6.4. For $(G, w)$ any $w$-basic group but $W_{2^{i}}$, the injection of a maximal cyclic group gives an isomorphism of Bockstein spectral sequences from $E_{2}$ on.

Remark A.1.6.5. The structure of $E_{*}\left(W_{2^{i+1}}\right),(* \leq 2)$ is given by

$$
E_{2}\left(W_{2^{i+1}}\right)=E\left(e_{3}\right) \otimes \mathrm{F}_{2}\left[\kappa_{4}\right]\left(1+e_{1}+c e_{1}\right),
$$

and $\beta_{2^{i-1}}\left(e_{1}\right)=c e_{1}, \beta_{2^{i-1}}\left(e_{3}\right)=0$, so $E_{i}=E\left(e_{3}\right) \otimes F_{2}\left[\kappa_{4}\right]$. Here, $\beta_{2^{i}}\left(e_{3}\right)=\kappa_{4}$, so $E_{i+1}=E_{\infty}=0$.

Lemma A.1.6.6. For $M_{16}$ the inclusion $Q_{8}=\langle x y, y z\rangle \subset M_{16}$ induces an isomorphism of Bockstein spectral sequences from $E_{2}$ on.

(The proofs of these lemmas are all the same. One looks at the formulae in $\S \mathrm{A} .1 .2$ for $H^{*}\left(M_{16} ; \mathrm{F}_{2}\right)$, and in $\S \mathrm{A} .1 .5$ for the rest of the groups. One then ignores the polynomial algebra $F_{2}\left[\kappa_{4}\right]$, and calculates the $S q^{1}$-homology of the remaining terms. In each case the resulting homology group consists of single copies of $F_{2}$ in dimensions 0 and 3. The only remaining step is to check that the term in dimension 3 in fact restricts to the corresponding element in the stated subgroup. For every group but $M_{16}$ this is direct from the original names of the elements. For $M_{16}$ first observe, since $\mathbb{Z} / 4 \times \mathbb{Z} / 2$ is an index 2 subgroup, that a simple use of transfer-restriction implies that $8 \cdot H^{*}\left(B_{M_{16}} ; \mathbb{Z}\right)=0$. But since the quaternion group described in Lemma 4 has $\kappa_{4}$ an element of order 8, it must follow that the $e_{3}$ for $M_{16}$ maps nontrivially to the corresponding $e_{3}$ for $Q_{8}$.)

We turn to the proof of A.1.0.3, and proceed in order, checking the relevant groups in the table in Appendix 3. The arguments are very similar to those above, the cases $\mathbb{Z} / 2, \mathbb{Z} / 4^{-},\left(D_{2^{i}}, 1\right)$ being direct. The Bockstein spectral sequence for $\left(D_{2^{i}},(x, y) \rightarrow(1,-1)\right)$ is determined by its first Wu class, $e+f$. Thus

$$
S q_{w}^{1}(A)=S q^{1}(A)+A \cup(e+f) .
$$

From this

$$
E_{2}\left(D_{2^{i}}\right)=F_{2}\left[\kappa_{4}\right] e \oplus F_{2}\left[\kappa_{4}\right] w .
$$


Now, the subgroup $\langle x\rangle=\mathbb{Z} / 2^{i-1} \subset D_{2^{i}}$ has trivial orientation character, and it is easily checked that this gives an injection on cohomology.

Next are $D_{2^{i+1}, f_{1}}, D_{2^{i+1}, f_{2}}$ with Wu class $h$ in both cases. In both cases $E_{2}=0$, so the result follows from Lemma A.1.6.1.

The case $\left(S D_{2^{i}},(x, y) \rightarrow(-1,1)\right)$ is more delicate. Here the Wu class is $e$, and $E_{2}=F_{2}\left[\kappa_{4}\right]\left(e^{2}\right) \oplus F_{2}\left[\kappa_{4}\right]\left(e_{3}\right)$. But this implies that there is higher integral torsion in homology only in dimensions of the form $4 i+2$.

For $\left(S D_{2^{i}},(x, y) \rightarrow(-1,-1)\right)$ the Wu class is $c$ and $E_{2}=0$.

For $S D_{2^{i+1}, f_{1}} \cong D_{2^{i+1}, f_{1}}$ the Wu class is $e+f+h$ in the notation of A.1.5 since the proof of Lemma A.1.4.2 shows that

$$
S D_{2^{i+1}, f_{1}} \stackrel{\cong}{\rightrightarrows} D_{2^{i+1}, f_{1}}
$$

is given explicitly by $x \rightarrow x, y \rightarrow x y, z \rightarrow z$. We then can easily check for the $E_{2}$-term that $E_{2}\left(S D_{2^{i+1}, f_{1}}\right)=0$.

The case $\left(Q_{2^{i+1}, f_{2}}, h \rightarrow-1\right)$ is similar. Here again, using the isomorphism

$$
D_{2^{i+1}, f_{2}} \cong Q_{2^{i+1}, f_{2}},
$$

the Wu class is $e+f+h$, and as before $E_{2}=0$.

Finally, for $M_{16}$ the Wu class is $a$, and again $E_{2}=0$.

\section{APPENDIX 2}

THE STRUCTURE OF $L_{1}^{p}(\mathbb{Z}[s] \pi)$ FOR $\pi$ A FINITE 2-GROUP

Let $\mathbb{Z}[s]$ be the polynomial ring and $\mathbb{Z}[s] \pi$ the group ring with involution $\tau(s)=1-s, \tau(g)=g^{-1}$ for $g \in \pi$. The groups $L_{*}^{p}(\mathbb{Z}[s] \pi)$ and $L_{*}^{h}(\mathbb{Z}[s] \pi)$ were studied in $[\mathrm{M}-\mathrm{O}]$. Essentially complete answers were given except for $L_{1}^{p}(\mathbb{Z}[s] \pi)$ and functors of representations of the form $M_{n}(\mathbb{Q}\langle-1,-1\rangle)$. We will complete the analysis given in $[\mathrm{M}-\mathrm{O}]$ here, for $L_{1}^{p}(\mathbb{Z}[s] \pi)$.

We recall some notation from [M-O].

$$
R=\underline{\lim }\left(\mathbb{Z} / 2^{l}[s]\right)=\left(\mathbb{Z}[s]^{\wedge}\right)_{2}
$$

is the completion of $\mathbb{Z}[s]$ at 2 . Since $\mathbb{Z}[s]$ has infinite rank over $\mathbb{Z}$ it follows that $\hat{\mathbb{Z}}_{2}[s]$ is properly contained in $\left(\mathbb{Z}[s]^{\wedge}\right)_{2}$, but it is also easily checked that $R$ is an integral domain. Next set

$$
S=R(1 / 2)
$$

$S$ is not a field but it is a P.I.D.

Lemma A.2.3. There is a Mayer-Vietoris exact sequence

$$
\cdots \rightarrow L_{n}^{p}(\mathbb{Z}[s] \pi) \rightarrow L_{n}^{p}(R \pi) \oplus L_{n}^{h}(\mathbb{Z}[s, 1 / 2] \pi) \rightarrow L_{n}^{h}(S \pi) \stackrel{\partial}{\rightarrow} \cdots .
$$

Proof. See [M-O, 5.5] 
The main result of this appendix is then

Theorem A.2.4. Let $\pi$ be a finite 2-group. Then there is an exact sequence

$$
0 \rightarrow \partial\left(L_{2}^{h}(S \pi)\right) \rightarrow L_{1}^{p}(\mathbb{Z}[s] \pi) \rightarrow L_{1}^{h}(\mathbb{Q} \pi) \rightarrow 0
$$

Remark. If $\mathbb{Q} \pi$ has no factors of the form $M_{n}\left(\left\langle^{-1} \mathbb{Q}^{-1}\right\rangle\right)$, then $\partial\left(L_{2}^{h}(S \pi)\right)$ is completely determined in [M-O] and the sequence above splits.

We deduce the theorem from the following facts

(F1) $L_{1}^{p}(R \pi)=0$,

(F2) $L_{1}^{h}(\mathbb{Z}[s, 1 / 2] \pi) \cong L_{1}^{h}(\mathbb{Q} \pi)\left(\right.$ here $\left.s \rightarrow \frac{1}{2}\right)$,

(F3) $L_{1}^{h}(S \pi)=0$.

(F1) is just Theorem 5.7 of [M-O].

From [M-O, 3.12], since $\mathbb{Z}[1 / 2] \pi$ is a regular ring for a finite 2-group, we have

$$
L_{1}^{h}(\mathbb{Z}[s, 1 / 2] \pi) \cong L_{1}^{h}(\mathbb{Z}[1 / 2] \pi) .
$$

By examining the localization exact sequence one sees that

$$
L_{1}^{h}(\mathbb{Z}[1 / 2] \pi) \stackrel{\approx}{\rightarrow} L_{1}^{h}(\mathbb{Q} \pi)
$$

which gives $(F 2)$. (In fact the localization exact sequence and devissage give an exact sequence

$$
\cdots \rightarrow \coprod_{p \text { odd }} L_{n+1}^{p}\left(\mathbf{F}_{p} \pi\right) \stackrel{\partial}{\rightarrow} L_{n}^{p}(\mathbb{Z}[1 / 2] \pi) \rightarrow L_{n}^{p}(\mathbb{Q} \pi) \rightarrow \coprod_{p \text { odd }} L_{n}^{p}\left(\mathbf{F}_{p} \pi\right) \rightarrow \cdots
$$

and the groups $L_{n}^{p}\left(\mathrm{~F}_{p} \pi\right)$ vanish for $p$ odd and $n \equiv 1,2$, or $3(\bmod 4)$. Then, one uses an easy comparison argument to pass from $L^{p}$ to $L^{h}$.)

With these preliminaries out of the way our main result is

Theorem A.2.6. $L_{1}^{h}(S \pi)=0$ for any finite 2-group $\pi$.

Proof. Consider the localization sequence

$$
\begin{aligned}
\cdots & \rightarrow L_{2}^{h}(Q S \pi) \oplus \coprod_{\wp} L_{2}^{h}\left(\widehat{S}_{\wp} \pi\right) \rightarrow \coprod_{\wp} L_{2}^{h}\left(\widehat{S}_{\wp}(1 / \wp) \pi\right) \\
& \rightarrow L_{1}^{h}(S \pi) \rightarrow L_{1}^{h}(Q S \pi) \oplus \coprod_{\wp} L_{1}^{h}\left(\widehat{S}_{\wp} \pi\right) \rightarrow \cdots,
\end{aligned}
$$

where $Q S$ is the quotient field and the $\wp$ run over all $\tau$-invariant primes in $S$. There are two things to note. First, it is not entirely obvious that the decorations above are all $h$ 's. But, since $\frac{1}{2} \in \widehat{S}_{\mathscr{P}}$ each of the rings $\widehat{S}_{\mathscr{P}}$ is a direct sum of maximal orders, easily seen to be either involution invariant or interchanged in pairs by the involution. Moreover, each summand contributes a copy of $\mathbb{Z}$ to $K_{0}\left(\widehat{S}_{\mathscr{C}} \pi\right)$. Second, from [M-O] primes are generated by irreducible monic polynomials $f \in \widehat{\mathbb{Z}}_{2}[s]$. Since $\tau$ acts nontrivially on $S /\langle f\rangle \cong \widehat{\mathbb{Q}}_{2}[s] /\langle f\rangle$, there are no ramified primes in $S$ over $S^{\tau}$. Then a direct application of [R 1 , Proposition 7.3, pp. 155] completes the demonstration that the $h$-decorations are correct. 
We have $L_{*}^{h}\left(\widehat{S}_{\wp} \pi\right) \cong L_{*}^{h}(S / \wp \pi)$ for each $\tau$-invariant prime, and $S / \wp$ is a finite dimensional extension field of $\widehat{\mathbb{Q}}_{2}$. Moreover, because $\wp$ is nonsplit and nonramified $\tau$ restricted to $K=S / \wp$ is a nontrivial automorphism with fixed field $\mathrm{F}_{\wp} \subset K$ having index 2 .

Thus $K \pi=\amalg M_{n_{i}}\left(K_{i}\right)$ is simply a direct sum of matrix rings over finite extensions of $K$. If one of these matrix rings is fixed under $\tau$, then $\tau \mid K_{i}$ is nontrivial. Otherwise, the $M_{n_{i}}\left(K_{i}\right)$ are permuted in pairs by $\tau$, hence they will contribute nothing to $L_{*}^{h}\left(\widehat{S}_{\wp} \pi\right)$.

In the case of a $M_{n_{i}}\left(K_{i}\right)$ with $K_{i}$ acted on nontrivially by the involution we have

$$
L_{*}^{p}\left(M_{n_{i}}\left(K_{i}\right)\right) \cong L_{*}^{p}\left(K_{i}, \tau\right)= \begin{cases}\mathbb{Z} / 2 \oplus \mathbb{Z} / 2, & * \text { even }, \\ 0, & * \text { odd }\end{cases}
$$

the invariants being rank and discriminant. Consequently, passing to $L_{*}^{h}(S / \wp \pi)$ we obtain

$$
L_{*}^{h}(S / \wp \pi)= \begin{cases}V, & * \text { even }, \\ 0, & * \text { odd }\end{cases}
$$

with $V$ given by a split exact sequence $(\mathbb{Z} / 2)^{n(\wp)} \rightarrow V \rightarrow \mathbb{Z} / 2$ where $n(\wp)$ is the number of summands fixed under $\tau$. The elements of $(\mathbb{Z} / 2)^{n(\wp)}$ are detected by discriminants. The $\mathbb{Z} / 2$ quotient detects the rank.

Next, consider $Q S$, the quotient field of $S$. Since $Q S \pi$ is semisimple $L_{\text {odd }}^{p}(Q S \pi)=0$. We have the Ranicki-Rothenberg sequence

$$
\cdots \rightarrow L_{\mathrm{ev}}^{p}(Q S \pi) \rightarrow \widehat{H}^{\mathrm{ev}}\left(\mathbb{Z} / 2 ; \widetilde{K}_{0}(Q S \pi)\right) \rightarrow L_{\mathrm{odd}}^{h}(Q S \pi) \rightarrow L_{\text {odd }}^{p}(Q S \pi) \rightarrow \cdots
$$

Moreover it is clear that

$$
\partial: L_{0}^{p}(Q S \pi) \rightarrow \widehat{H}^{\mathrm{ev}}\left(\mathbb{Z} / 2 ; \widetilde{K}_{0}(Q S \pi)\right)
$$

is onto, and, since $1-2 s \in Q S$ is a unit, and $\tau(1-2 s)=-(1-2 s)$ it follows that $*\langle 1-2 s\rangle: L_{i}^{d}(Q S \pi) \leftrightarrow L_{i+2}^{d}(Q S \pi)$ is an isomorphism. Finally, since $\partial\langle 1-2 s\rangle$. $\theta=\partial \theta$, it follows that $L_{\text {odd }}^{h}(Q S \pi)=0$ as well. Thus, the localization sequence gives the exact sequence

$$
L_{2}^{h}(Q S \pi) \oplus \coprod_{\wp} L_{2}^{h}(S / \wp \pi) \rightarrow \coprod_{\wp} L_{2}^{h}\left(\widehat{S}_{\wp}(1 / \wp) \pi\right) \rightarrow L_{1}^{h}(S \pi) \rightarrow 0 .
$$

As our next step we must calculate $L_{2}^{h}\left(\widehat{S}_{\wp}(1 / \wp) \pi\right)$. Note that $\widehat{S}_{\wp}(1 / \wp)$ is a field with $\widehat{S}_{\wp}$ a discrete valuation ring and quotient field $S / \wp$. Moreover, since $\wp$ is not ramified $\wp$ is not a norm in $\widehat{S}_{\wp}$. By the general theory of complete local rings, if we set $\mathbb{F}_{\wp}=\left(\hat{S}_{\wp}(1 / \wp)\right)^{\tau}$ then

$$
\mathbb{F}_{\wp}^{\times} / N_{\tau}\left(\widehat{S}_{\wp}(1 / \wp)^{\times}\right)=\mathbb{Z} / 2 \oplus \mathbb{Z} / 2
$$

the first $\mathbb{Z} / 2$ generated by $\langle\wp\rangle$, and the second by an element $v$ in $\left(\widehat{S}_{\wp}\right)^{\tau}$ which maps to a nonnorm in the fixed field of $\widehat{S}_{\wp} / \wp$. Consequently, the associated 
$L$-group has order 16, and generators $\langle 1\rangle,\langle v\rangle,\langle\wp\rangle,\langle v \wp\rangle$. In (A.2.9) the image of the corresponding summand in $L_{2}^{h}(S / \wp \pi)$ is the subgroup generated by $\langle 1\rangle$ and $\langle v\rangle$, at least in the case where the summand is a matrix ring $M_{n_{i}}\left(Q S_{i}\right)$, but, since $\wp$ is invariant and nonramified, it is easy to check that every summand in $\widehat{S}_{\wp}(1 / \wp) \pi$ is a matrix ring of the correct form. Thus, we must show the remaining two generators are in the image from $L_{2}^{h}(Q S \pi)$, at least modulo the image of $L_{2}^{h}(S / \wp \pi)$.

Note in particular that the quotient

$$
L_{2}^{h}\left(\widehat{S}_{\wp}(1 / \wp) \pi\right) / \operatorname{im} L_{2}^{h}(\widehat{S} \pi)=L_{2}^{K}\left(\widehat{S}_{\wp}(1 / \wp) \pi\right) / \operatorname{im} L_{2}^{K}\left(\widehat{S}_{\wp} \pi\right) .
$$

It follows that we can apply Morita equivalence.

We first consider the case where the summand we are looking at has the form $M_{n_{i}}\left(Q S_{i}\right)$. By Morita theory we can assume $n_{i}=1$, so the matrix ring is the center. Since $\wp$ is principal, nonramified, a fairly direct argument shows that $\wp$ is actually represented by an involution invariant polynomial $p$ of finite degree $n$ in s. Moreover there is an element $\bar{v}$ of degree $<n$ which has image $v$ on factoring out by $\wp$. Then the image of $\langle p\rangle$ is exactly $\langle\wp\rangle$, while the image of $\langle\bar{v} p\rangle$ is $\langle\wp\rangle$ at $\wp$, and has nontrivial image only at other primes which divide $\bar{v}$. But these all, of necessity, have degree strictly $<n$, and the result follows by induction.

This takes care of all the representations in $Q S \pi$ except those of the form

$$
M_{n}\left(Q S \otimes\left\langle\begin{array}{c}
-1-1 \\
\mathbb{Q}
\end{array}\right)\right)
$$

since every other representation is just a matrix algebra over $Q S$. Once more,

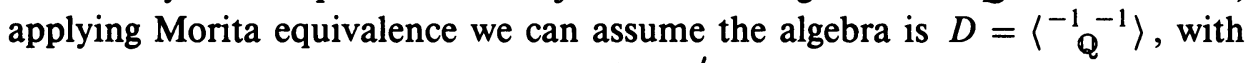
center $Q S$ and an appropriate involution $\tau^{\prime}$.

There is an invariant matrix $A \in M_{2}\left(K_{\varphi}\right)$ with $A^{2}=A$, and $\operatorname{det}(A)=0$ (just take a matrix Morita equivalent to $\left(\begin{array}{ll}1 & 0 \\ 0 & 0\end{array}\right)$.) Lift $A$ to $\tilde{A} \in S \otimes D$ so that it is invariant and a polynomial of degree $<\operatorname{degree}\left(K_{\wp} / \widehat{\mathbb{Q}}_{2}\right)$ in $s$. Then, $g=n r(\widetilde{A})$ is a polynomial of degree less than twice the degree of a generator $f$ for $\wp . g=\operatorname{det}(A)=0 \bmod p$, hence $g=f h$ with $\operatorname{deg}(h)<\operatorname{deg}(f)$. This class has image either $\langle\wp\rangle$ or $\langle 0 \wp\rangle$ modulo the previous elements, and elements of strictly lower degree. Moreover, if we multiply this element by the central element $\bar{v}$ we see easily that it hits the other element, again modulo primes of lower degree. Thus, an easy induction on degree shows that the map

$$
L_{2 *}(Q S \otimes D) \oplus \coprod_{\wp} L_{2 *}\left(\hat{S}_{\wp} \otimes D\right) \rightarrow \coprod_{\wp} L_{2 *}\left(\widehat{S}_{\wp}(1 / \wp) \otimes D\right)
$$

is surjective, and we have proved that $L_{1}^{h}(S \pi)=0$. This completes the proof of the theorem. 
APPENDIX 3

TABLE OF TYPES FOR $w$-MODEL GROUPS

\begin{tabular}{|c|c|c|c|}
\hline Group & Character & Type & Representation \\
\hline$e$ & 1 & $O$ & $\mathbb{Q}$ \\
\hline $\mathbb{Z} / 2$ & 1 & $O$ & $\mathbb{Q}$ \\
\hline $\mathbb{Z} / 2$ & -1 & $G L$ & $\mathbb{Q}$ \\
\hline $\mathbb{Z} / 2^{i}, i>1$ & 1 & $U$ & $\mathbb{Q}\left(\zeta_{2^{i}}\right)$ \\
\hline $\mathbb{Z} / 4$ & -1 & $O$ & $\mathbb{Q}(i)$ \\
\hline $\mathbb{Z} / 2^{i}, i>2$ & -1 & $U$ & $\mathbb{Q}\left(\zeta_{2^{i}}\right)$ \\
\hline$W_{2^{i+1}}, i>1$ & $z \mapsto-1$ & $U$ & $M_{2}\left(\mathbb{Q}\left(\zeta_{2^{i-1}}\right)\right)$ \\
\hline$D_{2^{i}}, i>3$ & 1 & $O$ & $M_{2}\left(\mathbb{Q}\left(\lambda_{2^{i-1}}\right)\right)$ \\
\hline$D_{2^{i}}, i>3$ & $(x, y) \mapsto(1,-1)$ & $S p$ & $M_{2}\left(\mathbb{Q}\left(\lambda_{2^{i-1}}\right)\right)$ \\
\hline$D_{2^{i}}, i>3$ & $(x, y) \mapsto(-1,1)$ & $U$ & $M_{2}\left(\mathbb{Q}\left(\lambda_{2^{i-1}}\right)\right)$ \\
\hline$D_{2^{i}}, i>3$ & $(x, y) \mapsto(-1,-1)$ & $U$ & $M_{2}\left(\mathbb{Q}\left(\lambda_{2^{i-1}}\right)\right)$ \\
\hline$D_{2^{i+1}, f_{2}}$ & $z \mapsto-1$ & $O$ & $M_{2}\left(\mathbb{Q}\left(\zeta_{2^{i}}\right)\right)$ \\
\hline$\overline{D_{2^{i+1}, f_{1}}}$ & $z \mapsto-1$ & $O$ & $M_{4}\left(\mathbb{Q}\left(\lambda_{2^{i-1}}\right)\right)$ \\
\hline$S D_{2^{i}}$ & 1 & $U$ & $M_{2}\left(\mathbb{Q}\left(\eta_{2^{i-1}}\right)\right)$ \\
\hline$S D_{2^{\prime}}$ & $(x, y) \mapsto(1,-1)$ & $U$ & $M_{2}\left(\mathbb{Q}\left(\eta_{2^{i-1}}\right)\right)$ \\
\hline$S D_{2^{\prime}}$ & $(x, y) \mapsto(-1,1)$ & $O$ & $M_{2}\left(\mathbb{Q}\left(\eta_{2^{i-1}}\right)\right)$ \\
\hline$S D_{2^{\prime}}$ & $(x, y) \mapsto(-1,-1)$ & $S p$ & $M_{2}\left(\mathbb{Q}\left(\eta_{2^{i-1}}\right)\right)$ \\
\hline$S D_{2^{1+1}, f_{1}}$ & $z \mapsto-1$ & $S p$ & $M_{4}\left(\mathbb{Q}\left(\lambda_{2^{i-1}}\right)\right)$ \\
\hline$S D_{2^{i+1}, f_{2}}$ & $z \mapsto-1$ & $\bar{U}$ & $M_{2}\left(\mathbb{Q}\left(\zeta_{2^{i}}\right)\right)$ \\
\hline$S D_{2^{1+1}, f_{3}}$ & $z \mapsto-1$ & $O$ & $M_{2}\left(\mathbb{Q}\left(\left\langle Q_{\left(\lambda_{2}{ }^{\prime}-2\right)}^{-1,-1}\right\rangle\right)\right)$ \\
\hline$Q_{2^{i}}, i \geq 3$ & 1 & $S p$ & $\left\langle\begin{array}{c}-1,-1 \\
Q\left(\lambda_{2 i-2}\right)\end{array}\right\rangle$ \\
\hline$Q_{2^{i}}, i \geq 3$ & $(x, y) \mapsto(1,-1)$ & $O$ & $\left\langle\begin{array}{l}-1,-1 \\
\mathbf{Q}\left(\lambda_{2^{i}-2}\right)\end{array}\right\rangle$ \\
\hline$Q_{2^{i}}, i>3$ & $(x, y) \mapsto(-1,1)$ & $U$ & $\left\langle\begin{array}{r}-1,-1 \\
Q\left(\lambda_{2 i-2}\right)\end{array}\right\rangle$ \\
\hline$Q_{2^{i}}, i>3$ & $(x, y) \mapsto(-1,-1)$ & $O$ & $\left\langle\begin{array}{r}-1,-1 \\
\mathbf{Q}\left(\lambda_{2 i-2}\right)\end{array}\right\rangle$ \\
\hline$Q_{2^{i+1}, f_{1}}, i>2$ & $z \mapsto-1$ & $S p$ & $M_{2}\left(\left\langle\begin{array}{c}-1,-1 \\
\mathbf{Q}\left(\lambda_{2 i-2}\right)\end{array}\right)\right)$ \\
\hline$Q_{2^{1+1}, f_{2}}, i>1$ & $z \mapsto-1$ & $S p$ & $M_{2}\left(\mathbb{Q}\left(\zeta_{2^{\prime}}\right)\right)$ \\
\hline$M_{16}$ & $w,(3.7 \mathrm{c})$ & $O$ & $M_{2}(\mathbb{Q}(i))$ \\
\hline
\end{tabular}

Here $\zeta_{n}$ is a primitive $n$ th-root of $1, \lambda_{n}=\zeta_{n}+\zeta_{n}^{-1}$, and $\eta_{n}=\zeta_{n}-\zeta_{n}^{-1}$. 


\section{REFERENCES}

[B] W. Browder, Torsion in H-spaces, Ann. of Math. 74 (1961), 24-61.

[D] J. F. Davis, The surgery semicharacteristic, Proc. London Math. Soc. (3) 47 (1983), 411-428.

[D-R] J. F. Davis and A. Ranicki, Semi-invariants in surgery, $K$-Theory 1 (1987), 83-109.

[Di] T. Diethelm, The mod- $p$ cohomology rings of the non-abelian split metacyclic p-groups, Arch. Math. 44 (1985), 29-38.

[D-L] J. L. Dupont and G. Lusztig, On manifolds satisfying $\left(w_{1}\right)^{2}=0$, Topology 10 (1971), 183199.

[H-M-T-W] I. Hambleton, R. J. Milgram, L. Taylor, and B. Williams, Surgery with finite fundamental group, Proc. London Math Soc. (3) 56 (1988), 349-379.

[H-T-W] I. Hambleton, L. Taylor; and B. Williams, Detection theorems in $K$ and L-theory, preprint.

[K] M. A. Kervaire, Courbure intégrale généralisée et homotopie, Math. Ann. 131 (1956), 219-252.

[L] R. Lee, Semicharacteristic classes, Topology 12 (1973), 189-199.

[L-M-P] G. Lusztig, J. Milnor, and F. P. Peterson, Semicharacteristics and cobordism, Topology 8 (1969), 357-359.

[M-M] Ib Madsen, R. J. Milgram, The classifying spaces for surgery and cobordism of manifolds, Ann. of Math. Studies, no. 92, Princeton Univ. Press, 1979.

[M] R. J. Milgram, Surgery with finite fundamental group, The Lefschetz Centennial Conference, Part II, Compositio Math. 58 (1987), 75-88.

[M1] _ Surgery with finite fundamental group I: the obstructions, preprint, Stanford University, 1955.

[M2] __ Surgery with finite fundamental group II: the oozing conjecture, preprint, Stanford University, 1955.

[M-O] R. J. Milgram and R. Oliver, $P$-adic logarithms and the algebraic $K, L$ groups for certain transcendence degree 1 extension of $Z_{\pi}, J$. Pure Appl. Algebra (to appear).

[Mu] H. J. Munkholm, Mod 2 cohomology of $D 2^{n}$ and its extensions by $\mathbf{Z}_{2}$, Conf. on Algebraic Topology, (Univ. of Illinois at Chicago Circle, Chicago, Ill., 1968), pp. 234-252.

[R] A. Ranicki, Exact sequences in the algebraic theory of surgery, Math. Notes 26, Princeton, Univ. Press, 1981.

[R1] _ Localization in quadratic L-theory, Algebraic Topology, Waterloo 1978, Lecture Notes in Math., vol. 741, Springer-Verlag, 1979.

[Ru] D. J. Rusin, The mod-2 cohomology of metacyclic 2-groups, J. Pure Appl. Algebra 44 (1987), 315-327.

[S] R. E. Stong, Semi-characteristics and free group actions, Compositio Math. 29 (1974), 223 248.

Department of Mathematics, Stanford University, Stanford, California 94305

Department of Mathematics, Indiana University, Bloomington, Indiana 47405 